\title{
AGR-1 Irradiation Experiment Test Plan
}

October 2009
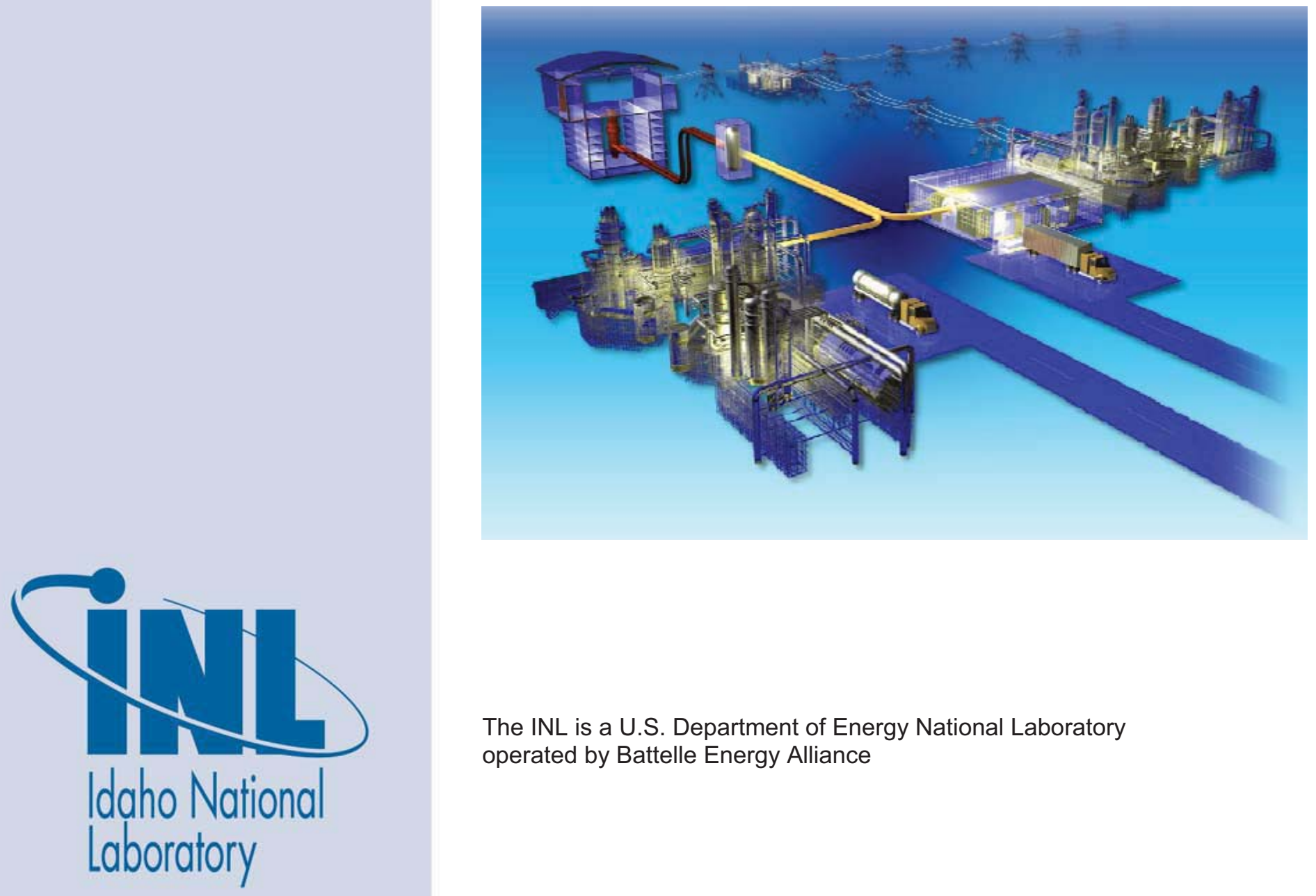

The INL is a U.S. Department of Energy National Laboratory operated by Battelle Energy Alliance 
INL/EXT-05-00593

Rev. 3

\section{AGR-1 Irradiation Experiment Test Plan}

October 2009

\section{Idaho National Laboratory \\ Idaho Falls, Idaho 83415}

http://www.inl.gov

Prepared for the

U.S. Department of Energy

Office of Nuclear Energy

Under DOE Idaho Operations Office

Contract DE-AC07-05ID14517 



\begin{tabular}{|l|ll|}
\hline \multirow{2}{*}{ AGR-1 Irradiation Experiment Test Plan } & Identifier: & INL/EXT-05-00593 \\
& Revision: & 3 \\
Effective Date: & $10 / 20 / 09$ & Page: i of viii \\
\hline
\end{tabular}

\begin{tabular}{|l|l|l}
\hline NGNP Project & Plan & eCR Number:
\end{tabular}

Prepared by:

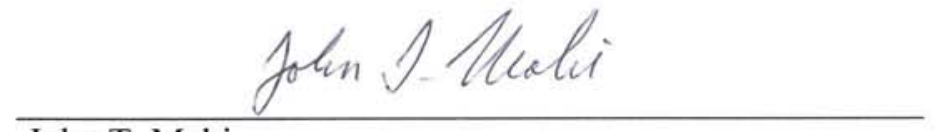

John T. Maki

\section{Approved by:}

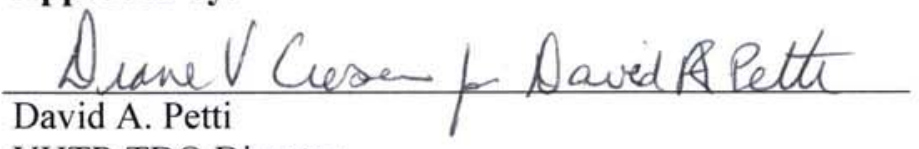

VHTR TDO Director

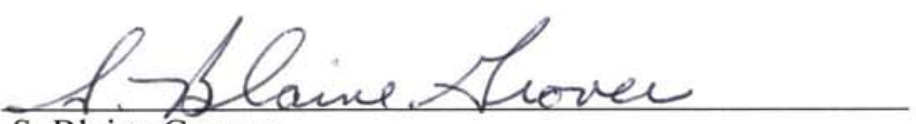
S. Blaine Grover

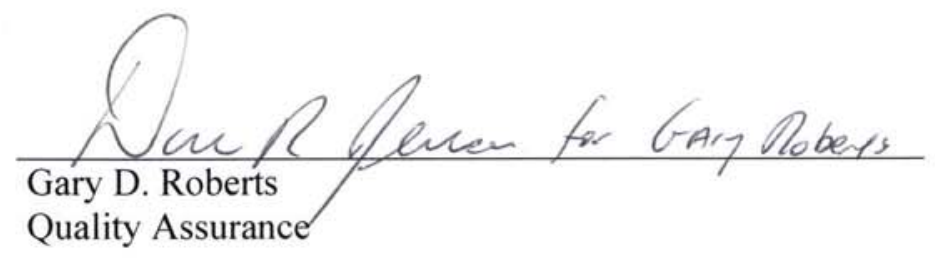

$\frac{10-20.09}{\text { Date }}$

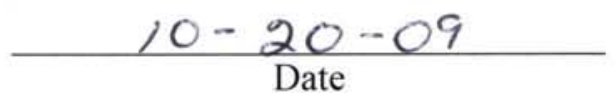

$\frac{10-20-09}{\text { Date }}$

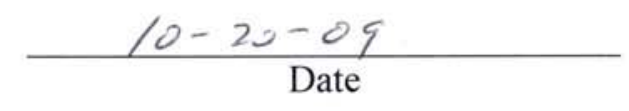


Identifier: INL/EXT-05-00593

Revision: $\quad 3$

Effective Date: $10 / 20 / 09 \quad$ Page: ii of viii

\section{REVISION LOG}

\begin{tabular}{|c|c|c|c|}
\hline Rev. & Date & Affected Pages & Revision Description \\
\hline 3 & $10 / 20 / 2009$ & All & Removed limited distribution, put in new format \\
\hline & & & \\
\hline & & & \\
\hline & & & \\
\hline & & & \\
\hline & & & \\
\hline & & & \\
\hline & & & \\
\hline & & & \\
\hline & & & \\
\hline & & & \\
\hline & & & \\
\hline & & & \\
\hline
\end{tabular}




\begin{tabular}{|c|c|c|}
\hline \multirow[t]{2}{*}{ AGR-1 Irradiation Experiment Test Plan } & $\begin{array}{l}\text { Identifier: } \\
\text { Revision: }\end{array}$ & $\begin{array}{l}\text { INL/EXT-05-00593 } \\
3\end{array}$ \\
\hline & Effective Date: & Page: iii of viii \\
\hline
\end{tabular}

\section{SUMMARY}

This document presents the current state of planning for the AGR-1 irradiation experiment, the first of eight planned irradiations for the Advanced Gas Reactor (AGR) Fuel Development and Qualification Program. Funding for this program is provided by the US Department of Energy (DOE) as part of the Next-Generation Nuclear Plant (NGNP) project. The objectives of the AGR-1 experiment are:

1. To gain experience with multi-capsule test train design, fabrication, and operation with the intent to reduce the probability of capsule or test train failure in subsequent irradiation tests.

2. To irradiate fuel produced in conjunction with the AGR fuel process development effort.

3. To provide data that will support the development of an understanding of the relationship between fuel fabrication processes, fuel product properties, and irradiation performance.

In order to achieve the test objectives, the AGR-1 experiment will be irradiated in the B-10 position of the Advanced Test Reactor (ATR) at the Idaho National Laboratory (INL). The test will contain six independently controlled and monitored capsules. Each capsule will contain a single type, or variant, of the AGR coated fuel. The irradiation is planned for about 725 effective full power days (approximately 2.5 calendar years) with a time-averaged, volume-average temperature ranging between approximately 980 and $1080{ }^{\circ} \mathrm{C}$. Average fuel burnup, for the entire test, will be greater than $18 \%$ FIMA, and the fuel will experience fast neutron fluences between approximately 2.5 and $4.6 \times 10^{25} \mathrm{n} / \mathrm{m}^{2}(\mathrm{E}>0.18 \mathrm{MeV})$. 


\begin{tabular}{|l|lll|}
\hline \multirow{2}{*}{ AGR-1 Irradiation Experiment Test Plan } & Identifier: & INL/EXT-05-00593 \\
& Revision: & 3 & \\
& Effective Date: & $10 / 20 / 09$ & Page: iv of viii \\
\hline
\end{tabular}




\begin{tabular}{|l|lll|}
\hline \multirow{2}{*}{ AGR-1 Irradiation Experiment Test Plan } & Identifier: & INL/EXT-05-00593 \\
& Revision: & 3 & \\
& Effective Date: & $10 / 20 / 09$ & Page: v of viii \\
\hline
\end{tabular}

\section{CONTENTS}

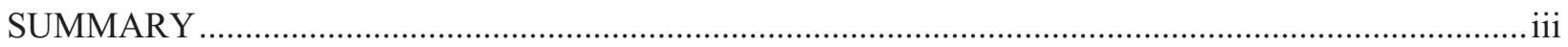

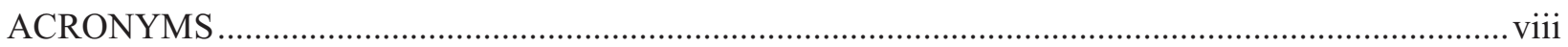

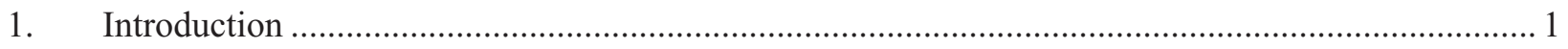

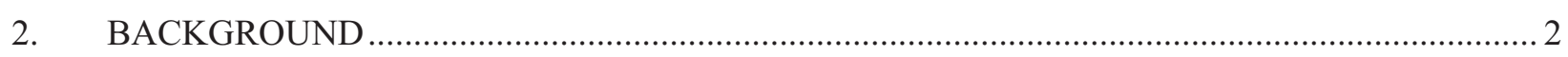

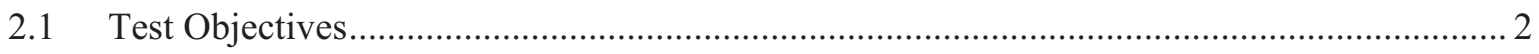

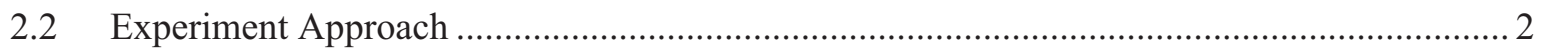

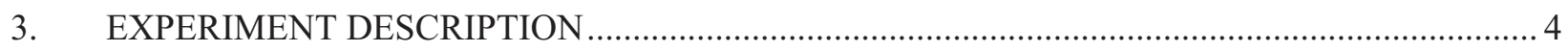

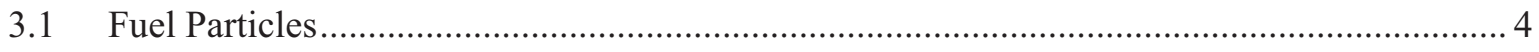

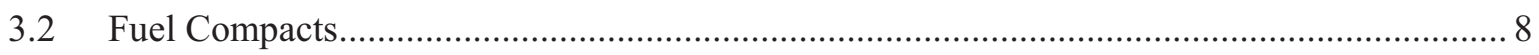

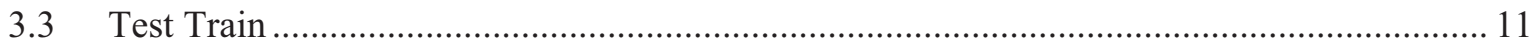

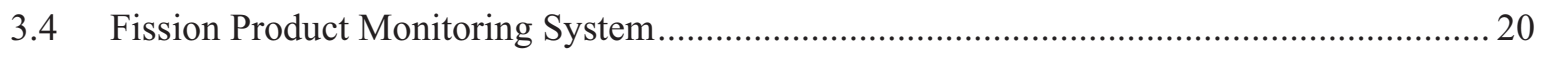

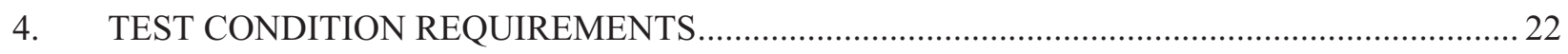

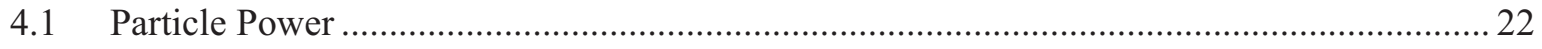

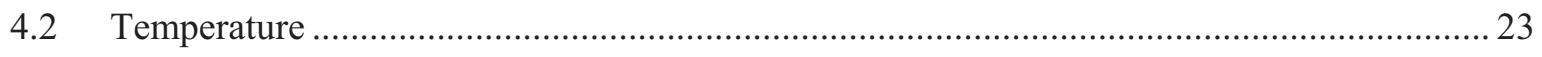

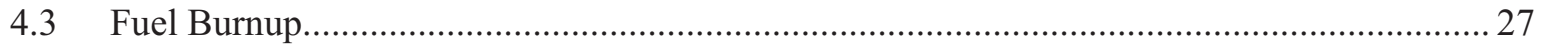

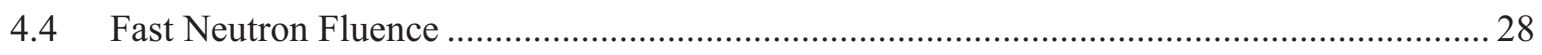

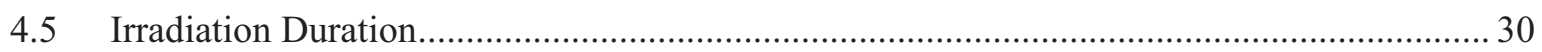

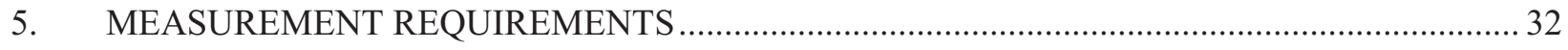

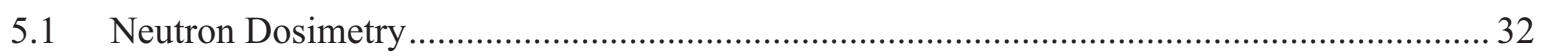

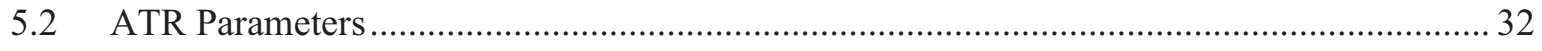

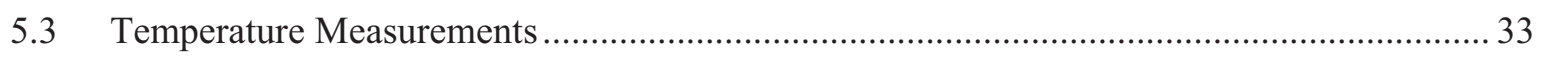

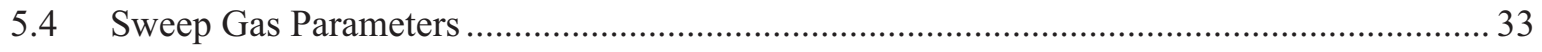

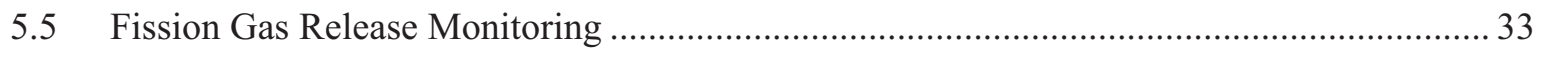

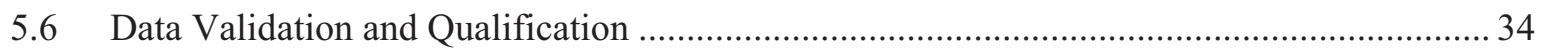

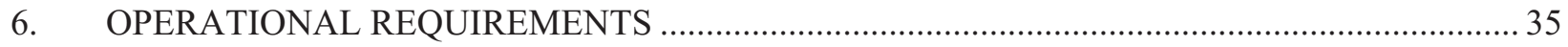

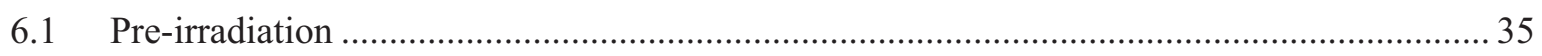

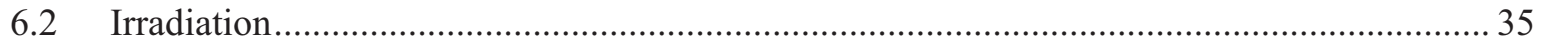

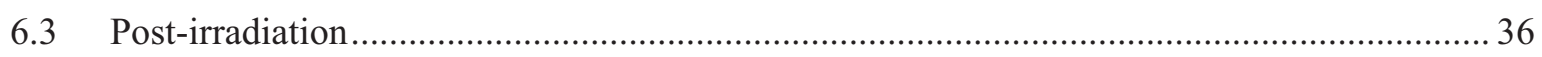

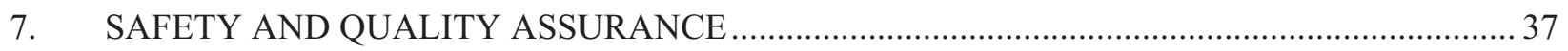

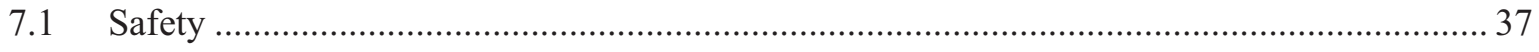

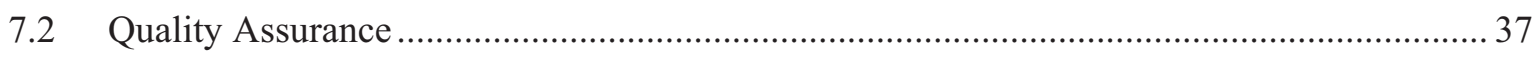

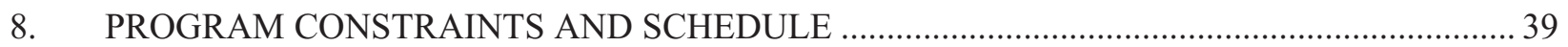




\begin{tabular}{|l|lll|}
\hline \multirow{2}{*}{ AGR-1 Irradiation Experiment Test Plan } & Identifier: & INL/EXT-05-00593 \\
& Revision: & 3 & \\
& Effective Date: & $10 / 20 / 09$ & Page: vi of viii \\
\hline
\end{tabular}

\section{FIGURES}

Figure 2-1. ATR core cross section displaying the B-10 position. .................................................. 3

Figure 3-1. Schematic of a typical TRISO-coated fuel particle............................................................. 4

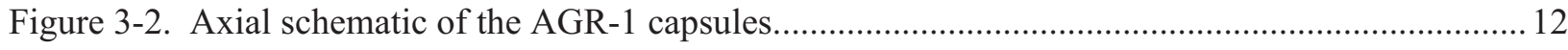

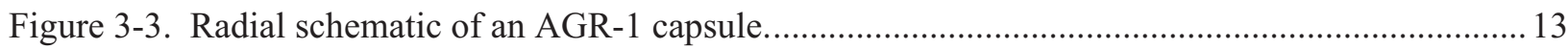

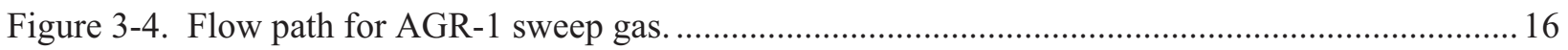

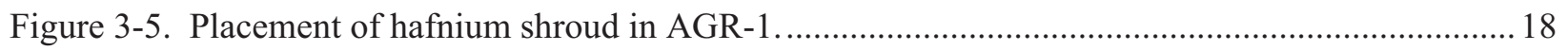

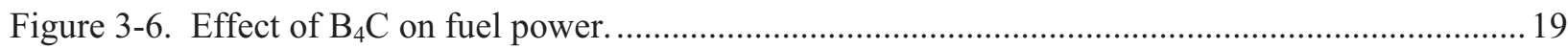

Figure 3-7. Effect of helium-3 sweep gas on fuel heat generation rate. ................................................ 19

Figure 3-8. Gross radiation monitor and spectrometer detector for one AGR-1 sweep gas line..............21

Figure 4-1. Compact average power for the maximum compact (Capsule 3 - Level 2 - Stack 1) and minimum compact (Capsule 6 - Level 4 - Stack 2)......................................................23

Figure 4-2. Thermal distributions in Capsule 3 fuel. ....................................................................... 24

Figure 4-3. Instantaneous peak temperature as a function of effective full power days (EFPD). ............25

Figure 4-4. Instantaneous volume-average temperature as a function of effective full power days

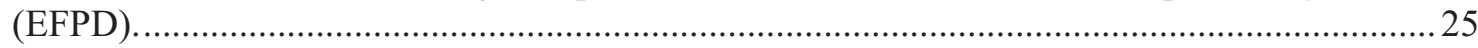

Figure 4-5. Time-average peak temperature as a function of EFPD (specification is $\leq 1250{ }^{\circ} \mathrm{C}$ )........... 26

Figure 4-6. Time-average, volume-average temperature as a function of EFPD (specification is

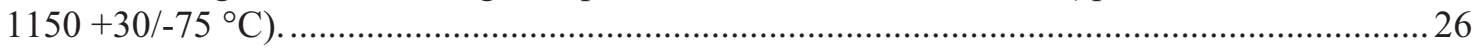

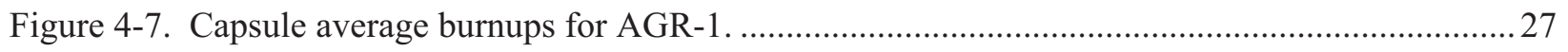

Figure 4-8. Compact average burnup for the maximum compact (Capsule 3 - Level 4 - Stack 1) and minimum compact (Capsule 6 - Level 4 - Stack 2) ......................................................28

Figure 4-9. Capsule average fast neutron fluences for AGR-1 ........................................................... 29

Figure 4-10. Compact average fast neutron fluence for the maximum compact (Capsule 4 - Level 2 - Stack 3) and minimum compact (Capsule 6 - Level 4 - Stack 2).

\section{TABLES}

Table 3-1. Primary functions of particle fuel components..................................................................... 4

Table 3-2. Selected properties for kernel Lot G73D-20-69302 …...................................................... 5

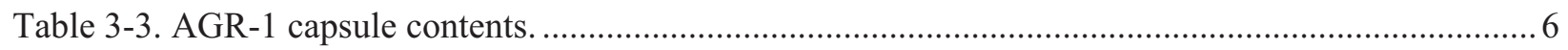

Table 3-4. Selected properties for AGR-1 coated particle composites..................................................... 7

Table 3-5. Selected properties for AGR-1 compacts. .......................................................................... 9

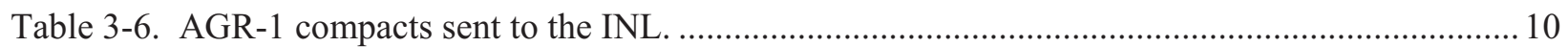




\begin{tabular}{|l|lll|} 
AGR-1 Irradiation Experiment Test Plan & Identifier: & INL/EXT-05-00593 \\
& Revision: & 3 & \\
& Effective Date: & $10 / 20 / 09$ & Page: vii of viii \\
\hline
\end{tabular}

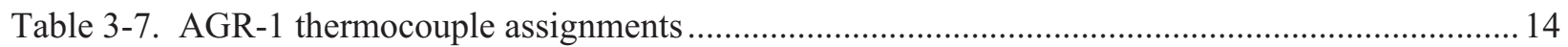

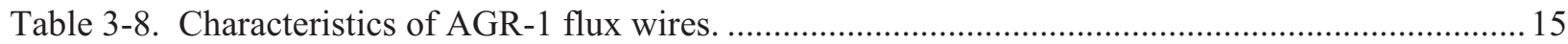

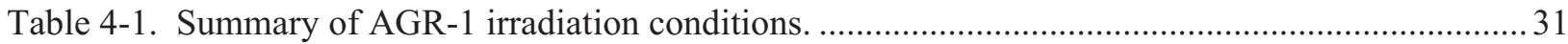

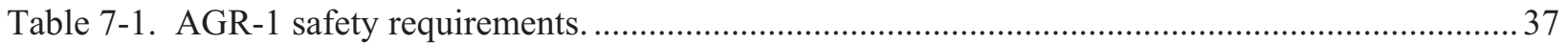




\begin{tabular}{|l|lll|}
\hline \multirow{2}{*}{ AGR-1 Irradiation Experiment Test Plan } & Identifier: & INL/EXT-05-00593 \\
& Revision: & 3 & \\
& Effective Date: & $10 / 20 / 09$ & Page: viii of viii \\
\hline
\end{tabular}

\section{ACRONYMS}

AGR

ATR

BWXT

DOE

EFPD

FIMA

FPMS

GT-MHR

HPGe

INL

IPyC

LEU

NGNP

$\mathrm{OPyC}$

ORNL

PALM

PIE

PyC

$\mathrm{R} / \mathrm{B}$

RMS

$\mathrm{SiC}$

TC

TRISO

UCO

VHTR
Advanced Gas Reactor

Advanced Test Reactor (INL)

BWX Technologies

Department of Energy (U.S.)

effective full power days

fissions in initial heavy metal atoms

fission product monitoring system

Gas-Turbine Modular Helium Reactor

hyper pure germanium

Idaho National Laboratory

inner pyrolytic carbon

low enriched uranium

Next Generation Nuclear Plant

outer pyrolytic carbon

Oak Ridge National Laboratory

powered axial locator mechanism

post irradiation examination

pyrolytic carbon

Release release rate to birth rate ratio

root mean square

silicon carbide

thermocouple

tri-isotropic

uranium oxycarbide

very-high-temperature gas-cooled reactor 


\begin{tabular}{|l|lll|}
\hline \multirow{2}{*}{ AGR-1 Irradiation Experiment Test Plan } & Identifier: & \multicolumn{2}{l|}{ INL/EXT-05-00593 } \\
& Revision: & 3 & \\
& Effective Date: & $10 / 20 / 09$ & Page: 1 of 41 \\
\hline
\end{tabular}

\section{Introduction}

Several fuel and material irradiation experiments are planned for the Advanced Gas Reactor (AGR) Fuel Development and Qualification Program which supports the development of the Very-HighTemperature gas-cooled Reactor (VHTR) under the Next-Generation Nuclear Plant (NGNP) project. The goals of these experiments (Petti, 2005) are to provide irradiation performance data to support fuel process development, to qualify fuel for normal operating conditions, to support development and validation of fuel performance and fission product transport models and codes, and to provide irradiated fuel and materials for post irradiation examination (PIE) and safety testing. AGR-1 is the first in this series of planned experiments to test tri-isotropic (TRISO)-coated, low enriched uranium (LEU) oxycarbide (UCO) fuel. This experiment is intended to serve as a shakedown test of a multi-capsule design to be used in subsequent irradiations and to test early variants of the fuel produced under this program.

This document presents the conceptual planning to implement requirements from the Technical Program Plan (Petti, 2005) and the Irradiation Test Specification (Maki, 2004) for the AGR-1 experiment. Following this introduction, the test objectives and experimental approach are outlined in Section 2; descriptions of the test articles, test train, and the fission product monitoring system are presented in Section 3; anticipated irradiation conditions including temperature, burnup, and fast neutron fluence are presented in Section 4; measurements associated with test conduct are described in Section 5; significant operational procedures that apply to AGR-1 are briefly described in Section 6; safety and quality assurance issues are outlined in Section 7; program constraints and test schedule are listed in Section 8; and finally, references are presented in Section 9. Requirements and planning associated with PIE and safety testing of the AGR-1 test articles will be presented elsewhere. 


\begin{tabular}{|c|lll|}
\hline \multirow{2}{*}{ AGR-1 Irradiation Experiment Test Plan } & Identifier: & INL/EXT-05-00593 \\
& Revision: & 3 & \\
& Effective Date: & $10 / 20 / 09$ & Page: 2 of 41 \\
\hline
\end{tabular}

\section{BACKGROUND}

\subsection{Test Objectives}

As defined in the AGR Technical Program Plan (Petti, 2005), the objectives of the AGR-1 experiment are:

1. To gain experience with multi-capsule test train design, fabrication, and operation with the intent to reduce the probability of capsule or test train failure in subsequent irradiation tests.

2. To irradiate fuel produced in conjunction with the AGR fuel process development effort.

3. To provide data that will support the development of an understanding of the relationship between fuel fabrication processes, fuel product properties, and irradiation performance.

The primary objective of the test is the shakedown of the test train. Should substantial failures occur during irradiation, such as leakage or malfunctions with temperature control, gas monitoring, or instrumentation, the test train could be removed from the reactor before the planned completion of the irradiation. Test components would then be examined to determine what modifications are required for subsequent test trains.

In conjunction with the fuel process development effort, AGR-1 will irradiate early fuel produced in a laboratory scale, small coater. Subsequent tests in the AGR program will irradiate fuel produced in a production scale, large coater. Also, as the VHTR design matures, the fuel design may change. A most likely design change will be an increase in fuel kernel diameter.

If successful, and the fuel is irradiated to a substantial burnup and fast neutron fluence, the test will provide irradiation performance data for baseline fuel and for variants of this fuel. Fuel variants will have been fabricated with predetermined, yet slight changes in selected fabrication processes. The test data will provide a link between fabrication processes, fuel product properties and irradiation performance. In addition to strengthening the fundamental understanding of this linkage, these data could be used to support the selection of the AGR reference fuel.

\subsection{Experiment Approach}

To achieve the test objectives outlined above, AGR-1 will be irradiated in the B-10 position of the Advanced Test Reactor (ATR) at the Idaho National Laboratory (INL). A core cross section indicating this location is displayed in Figure 2-1. Preliminary physics calculations (Chang, 2002) have shown that anticipated VHTR end of irradiation conditions (burnup up to about $20 \%$ FIMA and maximum fast neutron fluence of $5 \times 10^{25} \mathrm{n} / \mathrm{m}^{2}, \mathrm{E}>0.18 \mathrm{MeV}$ ) are best matched by the conditions obtained from irradiation in these large B positions, such as B-10, of the ATR. In addition, the rate of burnup and fast fluence accumulation, or acceleration, in these positions is less than three times that expected in the VHTR. Past US and German experience indicates that by keeping the acceleration factor under three, an irradiation test is more prototypic of an actual reactor irradiation (Petti et al., 2002). 


\begin{tabular}{|l|lll|}
\hline \multirow{2}{*}{ AGR-1 Irradiation Experiment Test Plan } & Identifier: & INL/EXT-05-00593 \\
& Revision: & 3 & \\
& Effective Date: & $10 / 20 / 09$ & Page: 3 of 41 \\
\hline
\end{tabular}

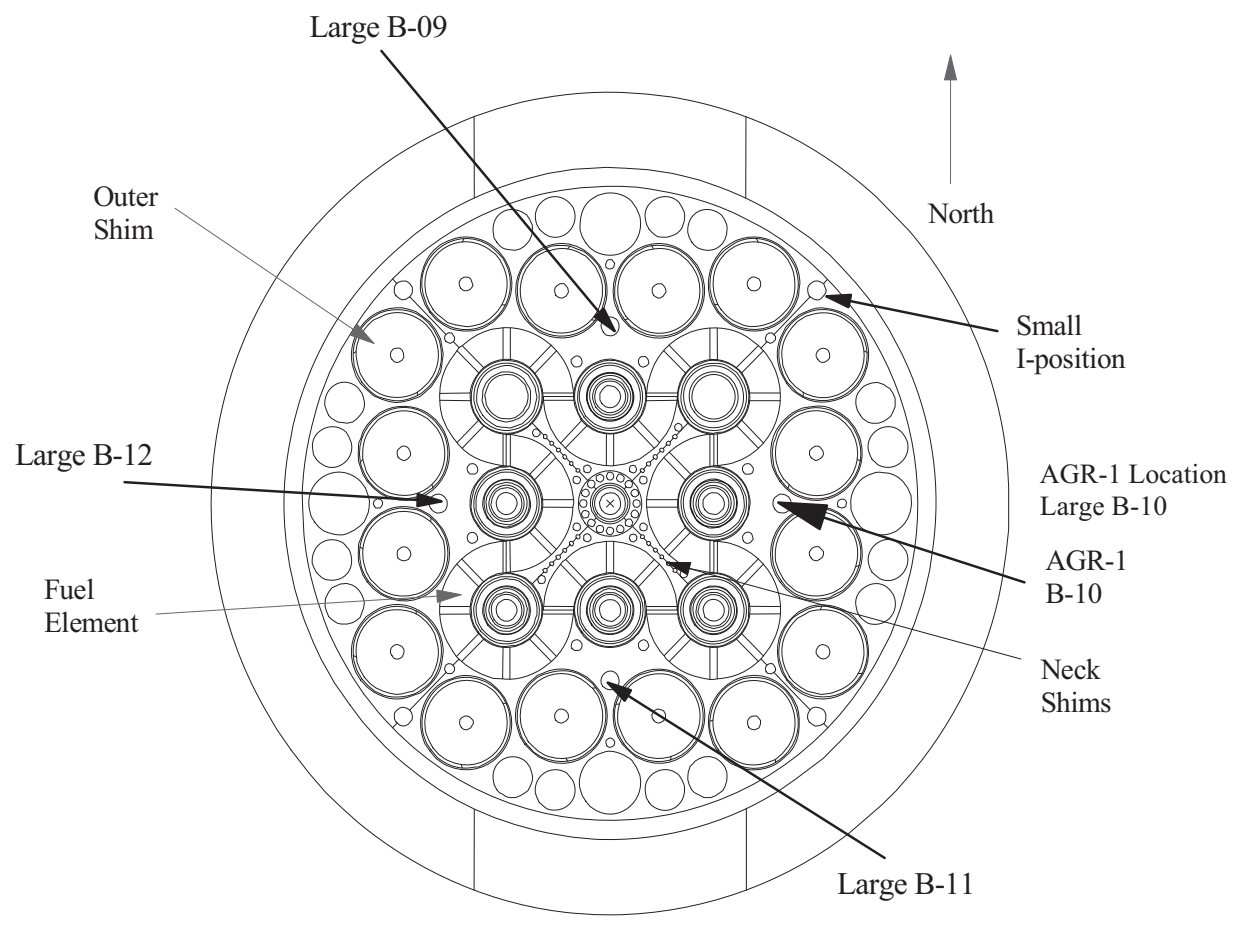

Figure 2-1. ATR core cross section displaying the B-10 position.

The test train planned for AGR-1 is based on the experience gained from previous irradiations in the ATR using instrumented lead experiments. Instrumented lead experiments are used for irradiations requiring a controlled environment and monitored parameters. The experiment test train positions the fuel within the test location and contains sweep gas lines and thermocouple wiring that is routed through access ports to external support systems.

The fuel to be irradiated in AGR-1 is based on the Gas-Turbine Modular Helium Reactor (GT-MHR) fissile particle design and fabricated, to the extent possible, according to German process practices. This fuel form was used as a starting point in defining a baseline fuel which is currently considered to possess the best attributes for successful irradiation in AGR-1. Three variant fuels will also be irradiated in AGR1. These variants will be fabricated according to predetermined variations in the baseline process and, as previously stated, provide new information concerning the linkage between fabrication processes, product properties and irradiation performance. 


\begin{tabular}{|l|lll|}
\hline \multirow{2}{*}{ AGR-1 Irradiation Experiment Test Plan } & Identifier: & \multicolumn{2}{l|}{ INL/EXT-05-00593 } \\
& Revision: & 3 & \\
& Effective Date: & $10 / 20 / 09$ & Page: 4 of 41 \\
\hline
\end{tabular}

\section{EXPERIMENT DESCRIPTION}

\subsection{Fuel Particles}

Fuel for AGR-1 will consist of TRISO- coated particles which are generally slightly less than $1 \mathrm{~mm}$ in diameter. Each particle has a central kernel containing the fuel material, a porous carbon buffer layer, an inner pyrolytic carbon (IPyC) layer, a silicon carbide (SiC) barrier coating, and an outer pyrolytic carbon (OPyC) layer. This fuel design is illustrated in Figure 3-1. The functions of each coating layer are listed in Table 3-1.

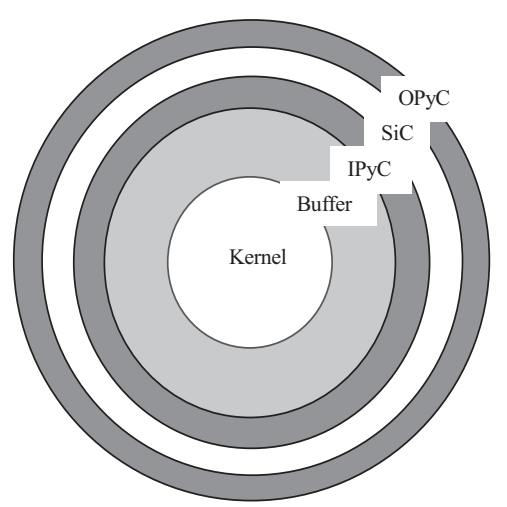

Figure 3-1. Schematic of a typical TRISO-coated fuel particle.

Table 3-1. Primary functions of particle fuel components.

\begin{tabular}{|l|l|}
\hline Component & Primary function \\
\hline Kernel & Contains fissile/fertile fuel \\
\hline Inner PyC & $\begin{array}{l}\text { Provides void space for fission product gases } \\
\text { and accommodates differential changes in } \\
\text { dimensions between coating layers and kernel }\end{array}$ \\
\hline SiC & $\begin{array}{l}\text { Structural layer which also protects the kernel } \\
\text { during SiC deposition }\end{array}$ \\
\hline Outer PyC & $\begin{array}{l}\text { Primary structural layer and primary fission } \\
\text { product barrier }\end{array}$ \\
\hline
\end{tabular}

Kernels for AGR-1 consist of LEU UCO fuel. The kernels were fabricated by BWX Technologies (BWXT) in accordance with the AGR-1 Fuel Product Specification (Barnes, 2006). Several production 


\begin{tabular}{|l|lll|}
\hline \multirow{2}{*}{ AGR-1 Irradiation Experiment Test Plan } & Identifier: & INL/EXT-05-00593 \\
& Revision: & 3 & \\
& Effective Date: & $10 / 20 / 09$ & Page: 5 of 41 \\
\hline
\end{tabular}

batches were combined into a single composite, Lot G73D-20-69302. This composite will be used in all of the AGR-1 fuel, including the baseline and each of the three fuel variants. Complete characterization data for this kernel lot are compiled in the Data Certification Package (BWXT, 2005). Selected kernel composite properties (from BWXT characterization except for kernel diameter and density which are from Oak Ridge National Laboratory (ORNL) characterization (Hunn and Lowden, 2006a)) and corresponding fuel product specifications are listed in Table 3-2.

Table 3-2. Selected properties for kernel Lot G73D-20-69302.

\begin{tabular}{|l|l|l|}
\hline Kernel Property & $\begin{array}{l}\text { Specified Range for } \\
\text { Mean Value }\end{array}$ & $\begin{array}{l}\text { Actual Mean Value } \pm \\
\text { Population Standard } \\
\text { Deviation }\end{array}$ \\
\hline Diameter $(\mu \mathrm{m})$ & $350 \pm 10$ & $349.7 \pm 9.0$ \\
\hline Density $\left(\mathrm{Mg} / \mathrm{m}^{3}\right)$ & $\geq 10.4$ & $10.924 \pm 0.015$ \\
\hline U-235 enrichment (wt \%) & $19.80 \pm 0.10$ & $19.736 \pm 0.047$ \\
\hline Carbon/uranium (atomic ratio) & $0.50 \pm 0.20$ & $0.3253 \pm 0.0028$ \\
\hline Oxygen/uranium (atomic ratio) & $1.50 \pm 0.20$ & $1.3613 \pm 0.0064$ \\
\hline$[$ Carbon + oxygen $] /$ uranium (atomic ratio) & $\leq 2.0$ & $1.6850 \pm 0.0093$ \\
\hline Total uranium (wt \%) & $\geq 87.0$ & $90.059 \pm 0.086$ \\
\hline Sulfur impurity(ppm - wt) & $\leq 1500$ & 608 \\
\hline All other impurities & various & $\begin{array}{l}\text { Below minimum } \\
\text { detection limits and } \\
\text { within specification }\end{array}$ \\
\hline
\end{tabular}

The UCO kernels were coated by ORNL who also provided characterization data (Hunn and Lowden, 2006a through 2006d). Coating was performed in accordance with the AGR-1 Fuel Product Specification (Barnes, 2006). Four particle composite lots will compromise the fuel to be irradiated in AGR-1. These fuels are briefly described below.

Baseline. Because of its excellent irradiation performance, coating process conditions used to fabricate historic German fuel were chosen as the starting point for the baseline fuel. Parametric studies refined these conditions for the specific coater to be used to coat AGR-1 fuel. This fuel is expected to perform successfully during irradiation.

Variant 1. The IPyC coating temperature will be increased relative to the baseline process for this variant. This change is expected to enhance the irradiation dimensional stability of the pyrocarbon but with increased uranium dispersion. Also, the IPyC layer density will be slightly lower than the baseline density.

Variant 2. The IPyC coating gas fraction will be increased relative to the baseline process for this variant. This change is also expected to enhance the irradiation dimensional stability of the 


\begin{tabular}{|l|lll|} 
AGR-1 Irradiation Experiment Test Plan & Identifier: & INL/EXT-05-00593 \\
& Revision: & 3 & \\
& Effective Date: & $10 / 20 / 09$ & Page: 6 of 41 \\
\hline
\end{tabular}

pyrocarbon but without significantly increasing uranium dispersion. Also, the IPyC layer density will be slightly higher than the baseline density.

Variant 3. The carrier gas composition for the $\mathrm{SiC}$ layer deposition will be changed from hydrogen to an argon-hydrogen mixture and deposition temperature will be lowered. This change is expected to reduce $\mathrm{SiC}$ defects resulting from uranium dispersion.

Each AGR-1 capsule will contain only one fuel type or variant. Baseline fuel will be irradiated in Capsules 6 and 3, Variant 1 in Capsule 5, Variant 2 in Capsule 2, and Variant 3 in Capsules 1 and 4. These assignments are listed in Table 3-3 where the capsules are numbered consecutively from the bottom, Capsule 1, to the top, Capsule 6.

A summary of selected properties, based on actual characterization data, for each of the four coated particle composites is listed in Table 3-4. Mean value specifications, where applicable, are also listed in Table 3-4 for comparison purposes.

Table 3-3. AGR-1 capsule contents.

\begin{tabular}{|c|c|c|}
\hline Location & Coated Particle Composite & Fuel Designation \\
\hline Capsule 6 & LEU01-46T & Baseline \\
\hline Capsule 5 & LEU01-47T & Variant 1 \\
\hline Capsule 4 & LEU01-49T & Variant 3 \\
\hline Capsule 3 & LEU01-46T & Baseline \\
\hline Capsule 2 & LEU01-48T & Variant 2 \\
\hline Capsule 1 & LEU01-49T & Variant 3 \\
\hline
\end{tabular}




\begin{tabular}{|l|lll|} 
AGR-1 Irradiation Experiment Test Plan & Identifier: & INL/EXT-05-00593 & \\
& Revision: & 3 & Page: 7 of 41 \\
\hline
\end{tabular}

Table 3-4. Selected properties for AGR-1 coated particle composites.

\begin{tabular}{|c|c|c|c|c|c|}
\hline \multirow[t]{2}{*}{ Property } & \multirow{2}{*}{$\begin{array}{l}\text { Specified } \\
\text { Range for } \\
\text { Mean Value }\end{array}$} & \multicolumn{4}{|c|}{ Actual Mean Value \pm Population Standard Deviation } \\
\hline & & Baseline & Variant 1 & Variant 2 & Variant 3 \\
\hline Buffer thickness $(\mu \mathrm{m})$ & $100 \pm 15$ & $103.5 \pm 8.2$ & $102.5 \pm 7.1$ & $102.9 \pm 7.3$ & $104.2 \pm 7.8$ \\
\hline IPyC thickness ( $\mu \mathrm{m})$ & $40 \pm 4$ & $39.4 \pm 2.3$ & $40.5 \pm 2.4$ & $40.1 \pm 2.8$ & $38.8 \pm 2.1$ \\
\hline SiC thickness $(\mu \mathrm{m})$ & $35 \pm 3$ & $35.3 \pm 1.3$ & $35.7 \pm 1.2$ & $35.0 \pm 1.0$ & $35.9 \pm 2.1$ \\
\hline OPyC thickness $(\mu \mathrm{m})$ & $40 \pm 4$ & $41.0 \pm 2.1$ & $41.1 \pm 2.4$ & $39.8 \pm 2.1$ & $39.3 \pm 2.1$ \\
\hline $\begin{array}{l}\text { Buffer density } \\
\left(\mathrm{Mg} / \mathrm{m}^{3}\right)\end{array}$ & $0.95 \pm 0.15$ & $1.10 \pm 0.04$ & $1.10 \pm 0.04$ & $1.10 \pm 0.04$ & $1.10 \pm 0.04$ \\
\hline IPyC density $\left(\mathrm{Mg} / \mathrm{m}^{3}\right)$ & $1.90 \pm 0.05$ & $1.904 \pm 0.014$ & $1.853 \pm 0.012$ & $1.912 \pm 0.015$ & $1.904 \pm 0.013$ \\
\hline SiC density $\left(\mathrm{Mg} / \mathrm{m}^{3}\right)$ & $\geq 3.19$ & $3.208 \pm 0.003$ & $3.206 \pm 0.002$ & $3.207 \pm 0.002$ & $3.205 \pm 0.001$ \\
\hline OPyC density $\left(\mathrm{Mg} / \mathrm{m}^{3}\right)$ & $1.90 \pm 0.05$ & $1.907 \pm 0.008$ & $1.898 \pm 0.009$ & $1.901 \pm 0.008$ & $1.911 \pm 0.008$ \\
\hline $\begin{array}{l}\text { IPyC anisotropy } \\
\text { (BAF) }\end{array}$ & $\leq 1.035$ & $1.022 \pm 0.002$ & $1.014 \pm 0.001$ & $1.023 \pm 0.002$ & $1.029 \pm 0.002$ \\
\hline $\begin{array}{l}\text { OPyC anisotropy } \\
\text { (BAF) }\end{array}$ & $\leq 1.035$ & $1.019 \pm 0.003$ & $1.013 \pm 0.002$ & $1.018 \pm 0.001$ & $1.021 \pm 0.003$ \\
\hline $\begin{array}{l}\text { IPyC anisotropy post } \\
\text { compact anneal (BAF) }\end{array}$ & $\begin{array}{l}\text { Not } \\
\text { specified }\end{array}$ & $1.033 \pm 0.004$ & $1.021 \pm 0.002$ & $1.036 \pm 0.001$ & $1.034 \pm 0.003$ \\
\hline $\begin{array}{l}\text { OPyC anisotropy post } \\
\text { compact anneal (BAF) }\end{array}$ & $\begin{array}{l}\text { Not } \\
\text { specified }\end{array}$ & $1.033 \pm 0.003$ & $1.030 \pm 0.003$ & $1.029 \pm 0.004$ & $1.036 \pm 0.002$ \\
\hline $\begin{array}{l}\text { Sphericity } \\
\text { (aspect ratio) }\end{array}$ & $\begin{array}{l}\text { Mean not } \\
\text { specified }^{(b)}\end{array}$ & $1.054 \pm 0.019$ & $1.056 \pm 0.019$ & $1.053 \pm 0.019$ & $1.055 \pm 0.018$ \\
\hline $\begin{array}{l}\text { Particle diameter }{ }^{(\mathrm{c})} \\
(\mu \mathrm{m})\end{array}$ & $\begin{array}{l}\text { Mean not } \\
\text { specified }\end{array}$ & 799.7 & 804.0 & 798.3 & 795.1 \\
\hline Particle mass (g) & $\begin{array}{l}\text { Mean not } \\
\text { specified }\end{array}$ & $7.27 \times 10^{-4}$ & $7.33 \times 10^{-4}$ & $7.24 \times 10^{-4}$ & $7.26 \times 10^{-4}$ \\
\hline
\end{tabular}

Notes: (a) Specification does not apply to Variants 1 and 2.

(b) Critical region is specified such that $\leq 1 \%$ of the particles shall have an aspect ratio $\geq 1.14$.

(c) Based upon mean average particle measurements, not sums of mean layer thicknesses. 


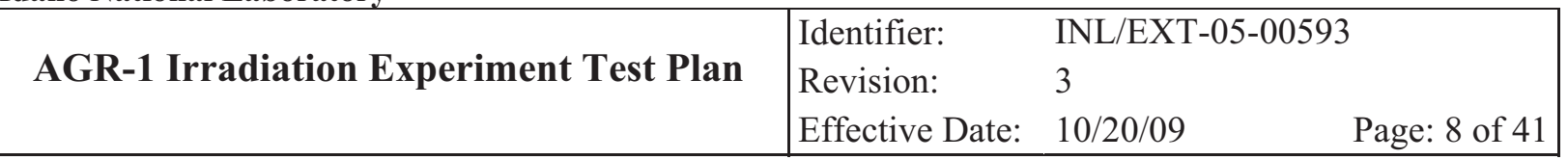

\subsection{Fuel Compacts}

After coating, AGR-1 fuel was formed into right cylindrical compacts. The compact matrix material is composed of a thermosetting carbonaceous material. Prior to compacting, the fuel particles were overcoated with approximately $165 \mu \mathrm{m}$ thick layers of the compact matrix material. This overcoat is intended to prevent particle-to-particle contact and help achieve the desired packing fraction of fuel particles.

AGR-1 compacts are nominally $25 \mathrm{~mm}$ in length and $12.3 \mathrm{~mm}$ in diameter. Future irradiations in the AGR program will consist of compacts nominally $49 \mathrm{~mm}$ in length. For AGR-1, the compacts are fabricated with fuel free end caps of matrix material approximately $1.5 \mathrm{~mm}$ thick. These end caps ensure smooth, protected surfaces that help to prevent fuel particle damage during handling. Although not done for AGR-1, a unique identifying serial number may be scribed into one end cap of each compact for future experiments.

The same compacting process was used for the baseline fuel and each fuel variant. A summary of selected properties, based on actual characterization data (Hunn et al., 2006a through 2006d) and derived from these data, for each fuel, type is listed in Table 3-5. Mean value specifications, where applicable, are also listed in Table 3-5 for comparison purposes. Data for compact mass, diameter and length are based on averages of those compacts sent to the INL. For traceability, Table 3-6 lists the compacts sent to the INL. 
Idaho National Laboratory

\begin{tabular}{|l|lll|} 
AGR-1 Irradiation Experiment Test Plan & Identifier: & INL/EXT-05-00593 & \\
& Revision: & 3 & Page: 9 of 41 \\
\hline
\end{tabular}

Table 3-5. Selected properties for AGR-1 compacts.

\begin{tabular}{|c|c|c|c|c|c|}
\hline \multirow[t]{2}{*}{ Property } & \multirow{2}{*}{$\begin{array}{l}\text { Specified } \\
\text { Range for } \\
\text { Mean Value }\end{array}$} & \multicolumn{4}{|c|}{ Actual Mean Value \pm Population Standard Deviation } \\
\hline & & Baseline & Variant 1 & Variant 2 & Variant 3 \\
\hline Compact mass $(\mathrm{g})$ & Not specified & 5.4789 & 5.3371 & 5.3736 & 5.5930 \\
\hline $\begin{array}{l}\text { Mean uranium loading } \\
\text { (g U/compact) }\end{array}$ & $0.905 \pm 0.04$ & 0.917 & 0.915 & 0.904 & 0.912 \\
\hline Diameter $^{(\mathrm{b})}(\mathrm{mm})$ & $12.22-12.46$ & $12.36 \pm 0.01$ & $12.36 \pm 0.01$ & $12.36 \pm 0.01$ & $12.34 \pm 0.01$ \\
\hline Length $^{(b)}(\mathrm{mm})$ & $25.02-25.40$ & $\begin{array}{l}25.066 \pm \\
0.080\end{array}$ & $\begin{array}{l}25.123 \pm \\
0.030\end{array}$ & $\begin{array}{l}25.077 \pm \\
0.065\end{array}$ & $\begin{array}{l}25.227 \pm \\
0.037\end{array}$ \\
\hline Number of particles per compact ${ }^{(a)}$ & Not specified & 4154 & 4145 & 4095 & 4132 \\
\hline $\begin{array}{l}\text { Particle volume packing fraction }{ }^{\text {(a) }} \\
(\%)\end{array}$ & Not specified & 36.99 & 37.42 & 36.26 & 36.04 \\
\hline $\begin{array}{l}\text { Effective overall compact density }{ }^{(a)} \\
\left(\mathrm{Mg} / \mathrm{m}^{3}\right)\end{array}$ & Not specified & 1.822 & 1.771 & 1.786 & 1.854 \\
\hline $\begin{array}{l}\text { Compact matrix density }{ }^{(a)} \\
\left(\mathrm{Mg} / \mathrm{m}^{3}\right)\end{array}$ & Not specified & 1.297 & 1.219 & 1.256 & 1.344 \\
\hline Compact weight $\% \mathrm{U}^{(\mathrm{a})}$ & Not specified & 16.737 & 17.144 & 16.823 & 16.306 \\
\hline Compact weight $\% \mathrm{O}^{\text {(a) }}$ & Not specified & 1.535 & 1.572 & 1.543 & 1.495 \\
\hline Compact weight $\% \mathrm{Si}^{\text {(a) }}$ & Not specified & 8.509 & 8.827 & 8.476 & 8.444 \\
\hline Compact weight $\% \mathrm{C}^{(\mathrm{a})}$ & Not specified & 73.219 & 72.457 & 73.158 & 73.754 \\
\hline $\begin{array}{l}\text { Iron content ( } \mu \mathrm{g} \text { Fe outside of } \\
\mathrm{SiC} / \text { compact) }\end{array}$ & $\leq 25$ & 1.57 & 0.97 & 1.88 & 1.81 \\
\hline $\begin{array}{l}\text { Chromium content ( } \mu \mathrm{g} \mathrm{Cr} \text { outside } \\
\text { of } \mathrm{SiC} / \text { compact) }\end{array}$ & $\leq 75$ & 1.58 & 1.62 & 0.50 & 0.18 \\
\hline $\begin{array}{l}\text { Manganese content ( } \mu \mathrm{g} \text { Mn outside } \\
\text { of } \mathrm{SiC} / \text { compact) }\end{array}$ & $\leq 75$ & 0.12 & 0.25 & 0.08 & 0.05 \\
\hline $\begin{array}{l}\text { Cobalt content ( } \mu \mathrm{g} \text { Co outside of } \\
\text { SiC/compact) }\end{array}$ & $\leq 75$ & 0.63 & 0.65 & 0.19 & 0.06 \\
\hline $\begin{array}{l}\text { Nickel content ( } \mu \mathrm{g} \text { Ni outside of } \\
\text { SiC/compact) }\end{array}$ & $\leq 75$ & 1.20 & 1.03 & 1.64 & 0.61 \\
\hline $\begin{array}{l}\text { Calcium content ( } \mu \mathrm{g} \text { Ca outside of } \\
\mathrm{SiC} / \text { compact) }\end{array}$ & $\leq 90$ & 7.95 & 5.86 & 15.13 & 10.10 \\
\hline $\begin{array}{l}\text { Aluminum content ( } \mu \mathrm{g} \mathrm{Al} \text { outside } \\
\text { of } \mathrm{SiC} / \text { compact) }\end{array}$ & $\leq 45$ & 8.54 & 8.21 & 11.21 & 7.46 \\
\hline $\begin{array}{l}\text { Titanium content ( } \mu \mathrm{g} \text { Ti outside of } \\
\text { SiC/compact) }\end{array}$ & Note (c) & 6.96 & 11.16 & 11.57 & 16.52 \\
\hline $\begin{array}{l}\text { Vanadium content ( } \mu \mathrm{g} \mathrm{V} \text { outside of } \\
\mathrm{SiC} / \text { compact) }\end{array}$ & Note (c) & 18.20 & 19.72 & 20.90 & 22.38 \\
\hline $\begin{array}{l}\text { U contamination fraction } \\
\text { (d) } \\
\text { (g exposed } \mathrm{U} / \mathrm{g} \mathrm{U} \text { in compact) }\end{array}$ & $\leq 1.0 \times 10^{-4}$ & 0 & 0 & 0 & 0 \\
\hline Defective SiC coating fraction ${ }^{(d)}$ & $\leq 2.0 \times 10^{-4}$ & $4.0 \times 10^{-5}$ & 0 & $2.0 \times 10^{-5}$ & 0 \\
\hline Defective IPyC coating fraction ${ }^{(\mathrm{d})}$ & $\leq 2.0 \times 10^{-4}$ & 0 & 0 & 0 & 0 \\
\hline Defective OPyC coating fraction ${ }^{(\mathrm{d})}$ & $\leq 1.0 \times 10^{-2}$ & 0 & $9.6 \times 10^{-4}$ & 0 & 0 \\
\hline
\end{tabular}

Notes: (a) Value derived from other characterized properties. 
Idaho National Laboratory

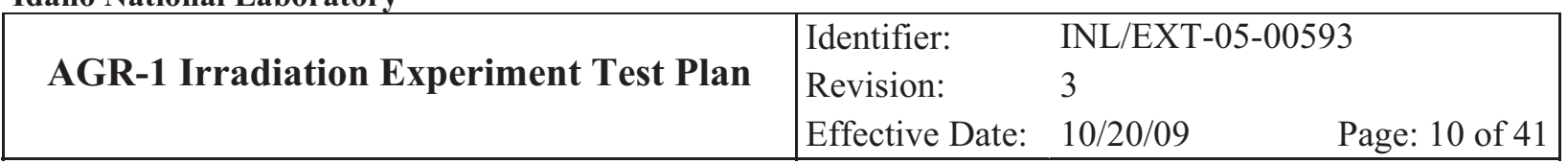

(b) Allowable range corresponding to upper and lower critical limits specified with no compacts exceeding the limits, which require $100 \%$ inspection of all compacts.

(c) Mean value specification of $\leq 400 \mu \mathrm{g}$ Ti plus V outside of SiC/compact.

(d) Value is an estimate of an attribute property, not the mean of a variable property.

Table 3-6. AGR-1 compacts sent to the INL.

\begin{tabular}{|c|c|c|c|c|c|c|c|}
\hline \multicolumn{2}{|c|}{$\begin{array}{c}\text { Baseline } \\
\text { Lot LEU01-46T-Z }\end{array}$} & \multicolumn{2}{|c|}{$\begin{array}{c}\text { Variant } 1 \\
\text { Lot LEU01-47T-Z }\end{array}$} & \multicolumn{2}{|c|}{$\begin{array}{c}\text { Variant } 2 \\
\text { Lot LEU01-48T-Z }\end{array}$} & \multicolumn{2}{|c|}{$\begin{array}{c}\text { Variant } 3 \\
\text { Lot LEU01-49T-Z }\end{array}$} \\
\hline $\begin{array}{l}\text { Compact } \\
\text { ID }\end{array}$ & $\begin{array}{l}\text { Assigned } \\
\text { Position }^{(a)}\end{array}$ & $\begin{array}{l}\text { Compact } \\
\text { ID }\end{array}$ & $\begin{array}{l}\text { Assigned } \\
\text { Position }^{(a)}\end{array}$ & $\begin{array}{l}\text { Compact } \\
\text { ID }\end{array}$ & $\begin{array}{l}\text { Assigned } \\
\text { Position }^{(a)}\end{array}$ & $\begin{array}{l}\text { Compact } \\
\text { ID }\end{array}$ & $\begin{array}{l}\text { Assigned } \\
\text { Position }^{(a)}\end{array}$ \\
\hline 23 & $3-1-1$ & 08 & $5-1-1$ & 27 & $2-1-1$ & 69 & $1-1-1$ \\
\hline 24 & $3-2-1$ & 13 & $5-2-1$ & 43 & $2-2-1$ & 54 & $1-2-1$ \\
\hline 39 & $3-3-1$ & 23 & $5-3-1$ & 03 & $2-3-1$ & 02 & $1-3-1$ \\
\hline 65 & $3-4-1$ & 79 & $5-4-1$ & 56 & $2-4-1$ & 77 & $1-4-1$ \\
\hline 12 & $3-1-2$ & 07 & $5-1-2$ & 15 & $2-1-2$ & 01 & $1-1-2$ \\
\hline 15 & $3-2-2$ & 56 & $5-2-2$ & 20 & $2-2-2$ & 03 & $1-2-2$ \\
\hline 36 & $3-3-2$ & 66 & $5-3-2$ & 38 & $2-3-2$ & 19 & $1-3-2$ \\
\hline 44 & $3-4-2$ & 74 & $5-4-2$ & 44 & $2-4-2$ & 28 & $1-4-2$ \\
\hline 17 & $3-1-3$ & 55 & $5-1-3$ & 58 & $2-1-3$ & 33 & $1-1-3$ \\
\hline 55 & $3-2-3$ & 65 & $5-2-3$ & 63 & $2-2-3$ & 34 & $1-2-3$ \\
\hline 56 & $3-3-3$ & 71 & $5-3-3$ & 66 & $2-3-3$ & 49 & $1-3-3$ \\
\hline 79 & $3-4-3$ & 72 & $5-4-3$ & 67 & $2-4-3$ & 52 & $1-4-3$ \\
\hline 32 & $6-1-1$ & 36 & Spare & 61 & Spare & 58 & $4-1-1$ \\
\hline 33 & $6-2-1$ & 38 & Spare & 34 & Spare & 64 & $4-2-1$ \\
\hline 49 & $6-3-1$ & 17 & Spare & 13 & Spare & 68 & 4-3-1 \\
\hline 53 & $6-4-1$ & 18 & Spare & 07 & Spare & 72 & $4-4-1$ \\
\hline 07 & $6-1-2$ & 77 & Spare & 46 & Spare & 73 & $4-1-2$ \\
\hline 09 & $6-2-2$ & 06 & Spare & 55 & Spare & 76 & $4-2-2$ \\
\hline 69 & $6-3-2$ & 20 & Spare & 19 & Spare & 44 & $4-3-2$ \\
\hline 77 & $6-4-2$ & 21 & Spare & 45 & Spare & 38 & $4-4-2$ \\
\hline 14 & $6-1-3$ & 33 & Spare & 60 & Spare & 14 & $4-1-3$ \\
\hline 30 & $6-2-3$ & 60 & Spare & 65 & Spare & 17 & $4-2-3$ \\
\hline 34 & $6-3-3$ & 04 & Spare & 02 & Spare & 36 & $4-3-3$ \\
\hline 76 & $6-4-3$ & 62 & Spare & 09 & Spare & 20 & 4-4-3 \\
\hline 42 & Spare & & & & & 16 & Spare \\
\hline 43 & Spare & & & & & 43 & Spare \\
\hline 57 & Spare & & & & & 24 & Spare \\
\hline 78 & Spare & & & & & 27 & Spare \\
\hline 05 & Spare & & & & & 66 & Spare \\
\hline 19 & Spare & & & & & 30 & Spare \\
\hline 52 & Spare & & & & & 29 & Spare \\
\hline 67 & Spare & & & & & 41 & Spare \\
\hline 46 & Spare & & & & & 18 & Spare \\
\hline 58 & Spare & & & & & 25 & Spare \\
\hline 60 & Spare & & & & & 37 & Spare \\
\hline 62 & Spare & & & & & 39 & Spare \\
\hline
\end{tabular}




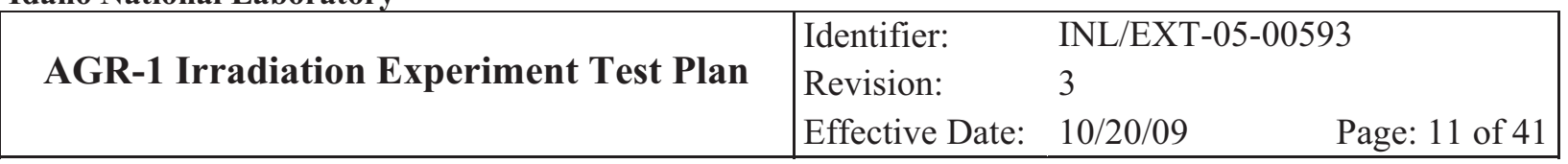

Notes: (a) Sequence is capsule number - level number - stack number where capsules are numbered sequentially from bottom (Capsule 1) to top (Capsule 6), levels are also numbered sequentially within a capsule from bottom (Level 1) to top (Level 4), and stacks are sequentially numbered clockwise from the northwest (Stack 1) to the southwest (Stack 3).

\subsection{Test Train}

As required by the Test Specification (Maki, 2004), the AGR-1 test train is a multi-capsule, instrumented lead experiment, designed for irradiation in the $38.1 \mathrm{~mm}$ (1.5 inch) diameter B-10 position of the ATR. The test train contains six capsules, each independently controlled for temperature and independently monitored for fission product gas release. An axial view of the test train is illustrated in Figure 3-2. Each AGR-1 capsule is $152.4 \mathrm{~mm}$ (6 inch) long and contains 12 fuel compacts arranged in three vertical stacks with each stack containing four compacts. Figure 3-3 illustrates a radial view of a capsule. Significant features of the test train are described below and further details are presented in the Technical and Functional Requirements documents (West et al., 2004; West et al., 2005).

\section{Thermocouples}

The selection process for thermocouples (TCs) was based on vendor data that indicated a mean time to failure for standard $\left(1 / 16\right.$ inch diameter) Type $\mathrm{N}$ or $\mathrm{K} \mathrm{TCs}$ at $1050{ }^{\circ} \mathrm{C}$ is six months. Considering this is within the range of expected sample holder temperatures and the test duration is expected to be on the order of two years, failure of standard TCs would seem likely. Therefore, accelerated furnace tests were conducted on commercial specialty TCs (with non-standard sheath or insulator materials) and INL fabricated Mo-Nb TCs that formed the basis for selection of the TCs with the greatest survival probability and least amount of drift.

Three $1.59 \mathrm{~mm}$ (1/16 inch) diameter TCs will be installed per capsule except for Capsules 1 (bottom) and 5, which will each contain two TCs and the top capsule (Capsule 6) which will contain two $1.59 \mathrm{~mm}$ diameter TCs and three $2.38 \mathrm{~mm}(3 / 32 \mathrm{inch})$ diameter TCs. (Capsule 5 was originally intended to contain three TCs, however, during fabrication of the test train, a braze failed in a Mo-Nb TC and was consequently, not installed.) Space constraints in the three thru tubes, which route the TCs and gas lines from top to bottom of the test train, limit the size and number of TCs that may be installed. The TCs will be placed in holes drilled in the graphite sample holders. Two capsules will have one TC located radially in the center with the other TCs located around the periphery. The remaining capsules will have all the TCs around the periphery. Avoidance of the hottest regions of the sample holders is expected to extend TC life. Therefore, only the INL produced Mo-Nb TCs, which are expected to have the greatest probability of survival, will be placed in the hotter portions of the capsules (center and western side of the capsule).

To avoid possible migration of $\mathrm{Ni}, \mathrm{Fe}$, or $\mathrm{Cr}$ from Inconel TC sheaths through the graphitic sample holders and compact matrix and potentially attack the SiC layer of the fuel, niobium sleeves will be placed around all Inconel sheathed TCs. These sleeves will cover the sheaths only within the graphite sample holders.

A summary of TC type, sheath and insulation materials, utilization of a protective $\mathrm{Nb}$ sleeve, and placement within the test train is provided in Table 3-7. 


\begin{tabular}{|l|lll|}
\hline \multirow{2}{*}{ AGR-1 Irradiation Experiment Test Plan } & Identifier: & \multicolumn{2}{l|}{ INL/EXT-05-00593 } \\
& Revision: & 3 & Page: 12 of 41 \\
\hline
\end{tabular}

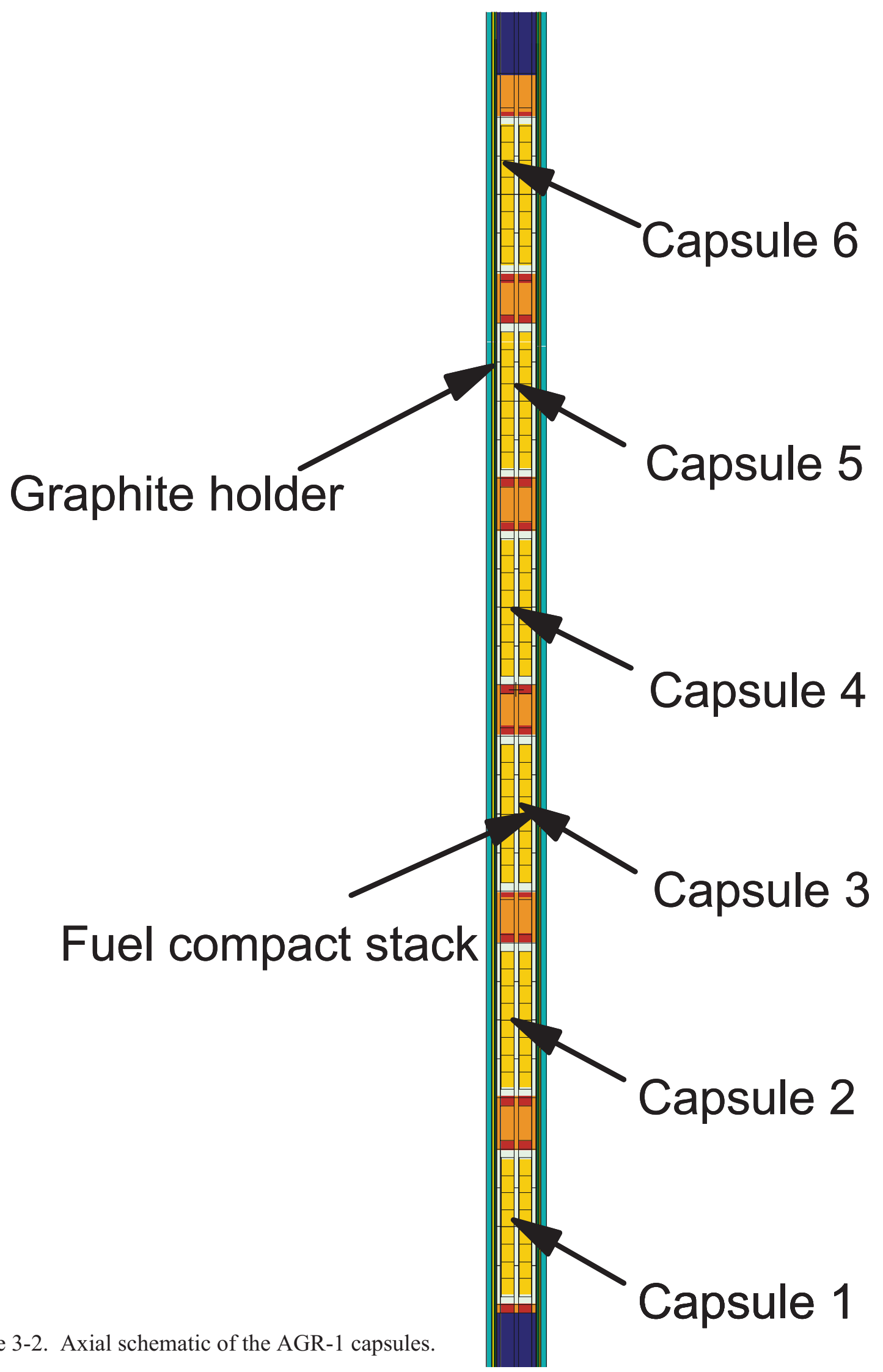

Figure 3-2. Axial schematic of the AGR-1 capsules. 


\begin{tabular}{|l|lll|}
\hline \multirow{2}{*}{ AGR-1 Irradiation Experiment Test Plan } & Identifier: & \multicolumn{2}{l|}{ INL/EXT-05-00593 } \\
& Revision: & 3 & \\
& Effective Date: & $10 / 20 / 09$ & Page: 13 of 41 \\
\hline
\end{tabular}

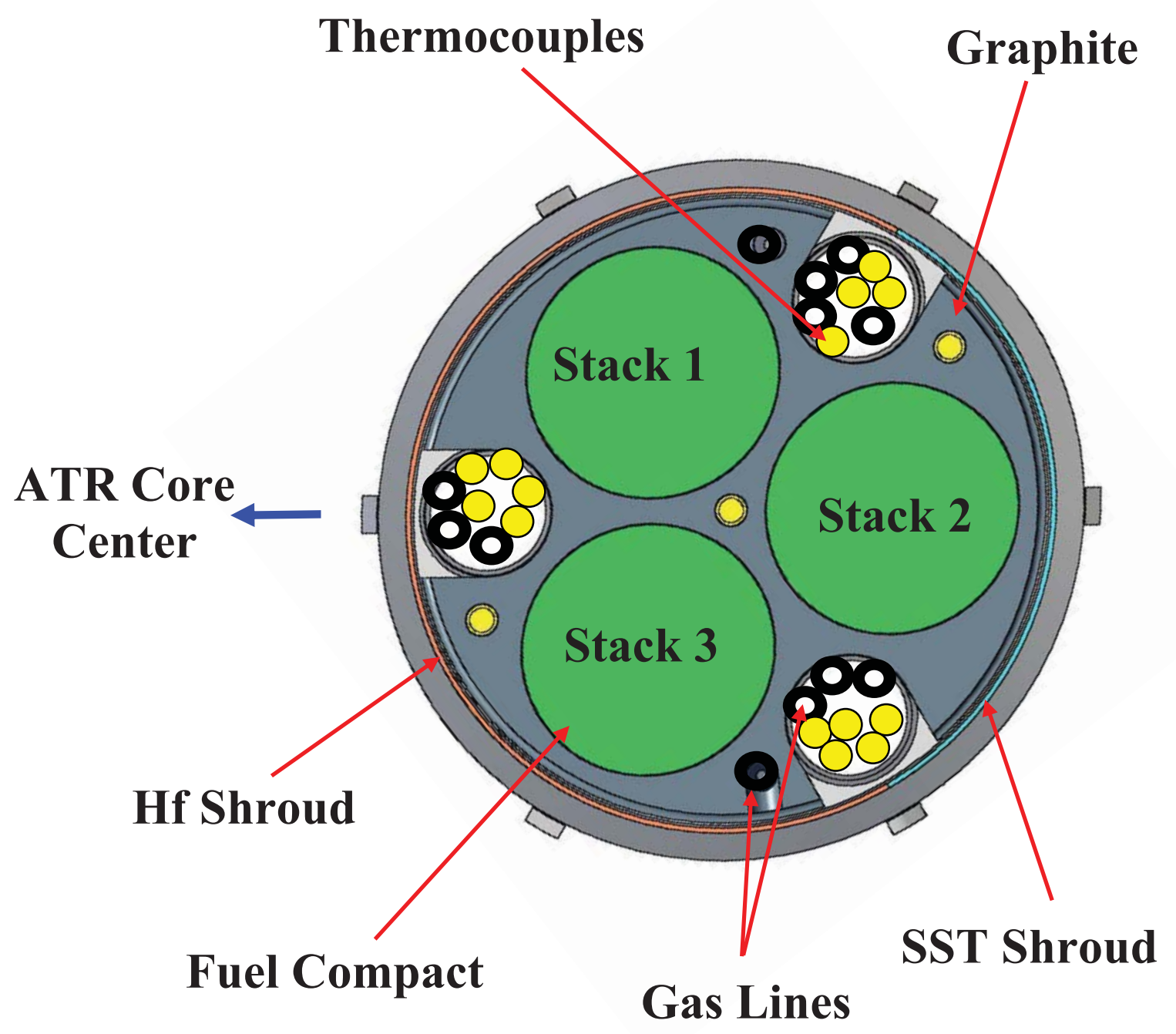

Figure 3-3. Radial schematic of an AGR-1 capsule. 


\begin{tabular}{|l|lll|} 
AGR-1 Irradiation Experiment Test Plan & Identifier: & INL/EXT-05-00593 \\
& Revision: & 3 & \\
& Effective Date: & $10 / 20 / 09$ & Page: 14 of 41 \\
\hline
\end{tabular}

Table 3-7. AGR-1 thermocouple assignments.

\begin{tabular}{|c|c|c|c|c|}
\hline Capsule & Location $^{(\mathrm{a})}$ & Thermocouple Type ${ }^{(b)}$ & Sheath / Insulation & Sleeve \\
\hline 6 & western & $\mathrm{Mo}-\mathrm{Nb}$ & $\mathrm{Nb}+0.1 \% \mathrm{Zr} / \mathrm{HfO}_{2}$ & none \\
\hline 6 & center & $\mathrm{Mo}-\mathrm{Nb}$ & $\mathrm{Nb}+0.1 \% \mathrm{Zr} / \mathrm{HfO}_{2}$ & none \\
\hline 6 & eastern & Type N (2.38 mm diameter) & $\mathrm{Mo} / \mathrm{Al}_{2} \mathrm{O}_{3}$ & none \\
\hline 6 & eastern & Type N (2.38 mm diameter) & $\mathrm{Mo} / \mathrm{Al}_{2} \mathrm{O}_{3}$ & none \\
\hline 6 & eastern & Type N (2.38 mm diameter) & $\mathrm{Mo} / \mathrm{Al}_{2} \mathrm{O}_{3}$ & none \\
\hline 5 & eastern & $\mathrm{Mo}-\mathrm{Nb}$ & $\mathrm{Nb}+0.1 \% \mathrm{Zr} / \mathrm{HfO}_{2}$ & none \\
\hline 5 & eastern & Type N & Inconel / MgO & $\mathrm{Nb}$ \\
\hline 4 & center & $\mathrm{Mo}-\mathrm{Nb}$ & $\mathrm{Nb}+0.1 \% \mathrm{Zr} / \mathrm{HfO}_{2}$ & none \\
\hline 4 & eastern & $\mathrm{Mo}-\mathrm{Nb}$ & $\mathrm{Nb}+0.1 \% \mathrm{Zr} / \mathrm{HfO}_{2}$ & none \\
\hline 4 & eastern & Type N & Inconel / MgO & $\mathrm{Nb}$ \\
\hline 3 & western & $\mathrm{Mo}-\mathrm{Nb}$ & $\mathrm{Nb}+0.1 \% \mathrm{Zr} / \mathrm{HfO}_{2}$ & none \\
\hline 3 & eastern & $\mathrm{Mo}-\mathrm{Nb}$ & $\mathrm{Nb}+0.1 \% \mathrm{Zr} / \mathrm{HfO}_{2}$ & none \\
\hline 3 & eastern & Type N & Inconel / MgO & $\mathrm{Nb}$ \\
\hline 2 & western & $\mathrm{Mo}-\mathrm{Nb}$ & $\mathrm{Nb}+0.1 \% \mathrm{Zr} / \mathrm{HfO}_{2}$ & none \\
\hline 2 & eastern & $\mathrm{Mo}-\mathrm{Nb}$ & $\mathrm{Nb}+0.1 \% \mathrm{Zr} / \mathrm{HfO}_{2}$ & none \\
\hline 2 & eastern & Type N & Inconel / MgO & $\mathrm{Nb}$ \\
\hline 1 & eastern & $\mathrm{Mo}-\mathrm{Nb}$ & $\mathrm{Nb}+0.1 \% \mathrm{Zr} / \mathrm{HfO}_{2}$ & none \\
\hline 1 & eastern & Type N & Inconel / MgO & $\mathrm{Nb}$ \\
\hline
\end{tabular}

Notes: (a) Western TCs are located in the outer portion of the graphite sample holder towards the reactor center. Eastern TCs are located in the outer portion of the graphite sample holder away from the reactor center. Center TCs are located in the center of the graphite sample holder.

(b) All TCs are $1.59 \mathrm{~mm}(1 / 16 \mathrm{inch})$ in diameter unless noted as $2.38 \mathrm{~mm}(3 / 32$ inch) in diameter.

\section{Melt Wires}

Each capsule will contain a beryllium melt wire encapsulated in a pure vanadium tube. The encapsulation will be about $7.9 \mathrm{~mm}$ long with an outer diameter of $1.3 \mathrm{~mm}$ and will be engraved with a unique identification number. The melt wires will be placed within holes drilled near the periphery and about midway up from the bottom of each graphite sample holder. Post irradiation examination of the melt wires will indicate if the capsule experienced temperatures in excess of $1287^{\circ} \mathrm{C}$.

\section{$\underline{\text { Neutron Monitors }}$}

In order to measure both thermal and fast neutron fluences, flux wires will be placed in each capsule. After irradiation, the induced activity of the wires will be converted to fluences with the appropriate neutron energy range and will also be used as a benchmark for physics analyses. Three materials will be used for the wires, pure $\mathrm{Fe}, \mathrm{V}+0.1 \% \mathrm{Co}$, and pure $\mathrm{Nb}$. Each wire will be encapsulated in a pure vanadium tube with an outer diameter of about $1.4 \mathrm{~mm}$. The lengths of the encapsulations will be about $7.1 \mathrm{~mm}$ for the Fe wire, $4.8 \mathrm{~mm}$ for the $\mathrm{V}+0.1 \%$ Co wire, and $8.6 \mathrm{~mm}$ for the $\mathrm{Nb}$ wire. A unique identification number will be engraved on each encapsulation. These encapsulated neutron monitors will be placed in holes drilled into the bottom and around the periphery of the graphite sample holders. Characteristics of the flux wires are listed in Table 3-8. 


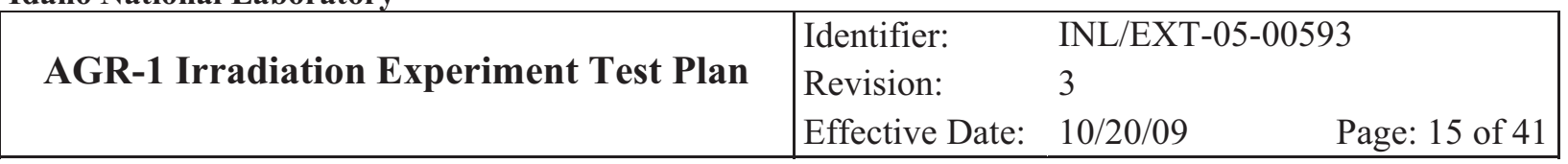

Table 3-8. Characteristics of AGR-1 flux wires.

\begin{tabular}{|l|l|l|l|}
\hline Material & Reaction & $\begin{array}{l}\text { Reaction Product } \\
\text { Half-Life }\end{array}$ & $\begin{array}{l}\text { Neutron Activation } \\
\text { Energy Range }\end{array}$ \\
\hline $\mathrm{V}+0.1 \% \mathrm{Co}$ & Co-59 $(\mathrm{n}, \gamma)$ Co-60 & 5.3 years & thermal \\
\hline $\mathrm{Fe}$ & Fe-54 (n,p) Mn-54 & 312 days & $1 \mathrm{MeV}$ threshold \\
\hline $\mathrm{Nb}$ & Nb-93 (n,n') Nb-93m & 16 years & $0.18 \mathrm{MeV}$ threshold \\
\hline
\end{tabular}

\section{$\underline{\text { Sweep Gas }}$}

Independent gas lines will route a mixture of helium and neon gases through each of the six capsules to provide temperature control and to sweep released fission product gases to the fission product monitoring system (FPMS). Temperature control is based upon temperature feedback from the TCs in each capsule and by varying the sweep gas composition (between $100 \%$ helium for high conductivity and $100 \%$ neon for low conductivity). This blending of sweep gases will be accomplished by a computerized mass flow controller before the gas enters the test train. Gas flow will be $\leq 50 \mathrm{sccm}$ (standard cubic centimeters per minute) at a pressure of about $15 \mathrm{psig}$ (pounds per square inch - gauge) or $0.103 \mathrm{MPa}-$ gauge. For high power reactor (powered axial locator mechanism [PALM]) cycles, the sweep gas may consist of $100 \% \mathrm{He}-3$. Actuation of this gas will be done manually and will flow at a pressure of about 200 psig (1.38 MPa-gauge). Use of He-3 for high power cycles is described further in the power shaping discussion below.

Sweep gas flow, originating from gas supply bottles, is routed to the gas verification panel where thermal conductivity and moisture measurements are performed for both the helium and neon gas lines. The gas then flows to the mass flow controller cabinet where the helium and neon gases are blended for each capsule and is then routed on to the capsule inlet isolation panel, which can be utilized to isolate inlet gas flow to each capsule independently during reactor outages or in the event of a failure. Upon exiting the capsule and test train, the gas flows through the outlet isolation panel to another panel containing a particulate filter, a moisture detector and a 3-way valve. The valve routes the gas either to the designated fission product monitor or the standby-backup fission product monitor. Another 3-way valve allows the gas to be routed to a manual grab sample line. After passing through the fission product monitor system, the gas lines combine into a common exhaust header that routes the gas through a tritium getter and a silver-zeolite filter. The exhaust gas is finally routed to the ATR stack. A schematic of this gas flow is presented in Figure 3-4. 


\begin{tabular}{|l|lll|}
\hline \multirow{2}{*}{ AGR-1 Irradiation Experiment Test Plan } & Identifier: & \multicolumn{2}{l|}{ INL/EXT-05-00593 } \\
& Revision: & 3 & \\
& Effective Date: & $10 / 20 / 09$ & Page: 16 of 41 \\
\hline
\end{tabular}

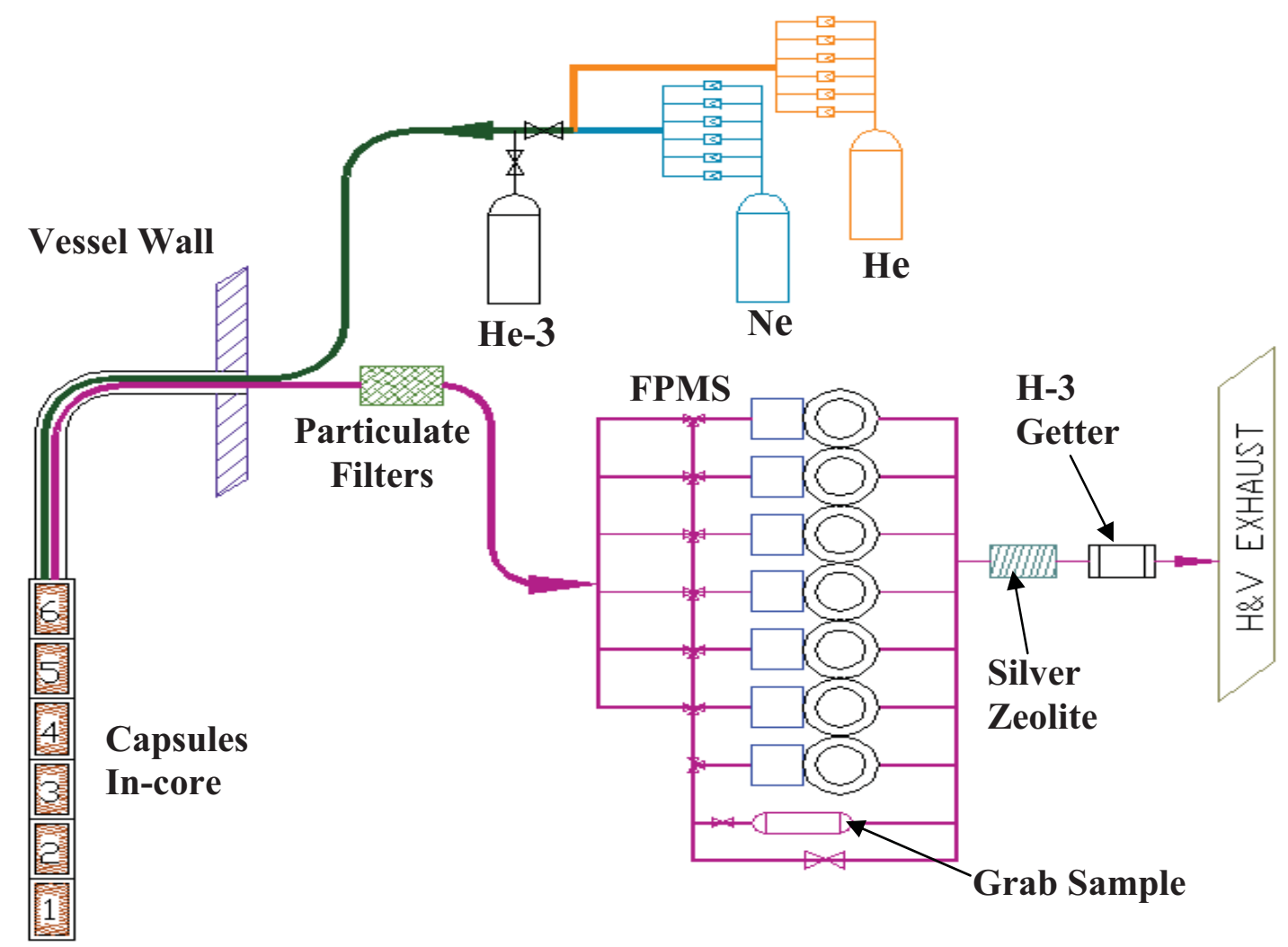

Figure 3-4. Flow path for AGR-1 sweep gas.

\section{$\underline{\text { Thru Tubes }}$}

Thru tubes route TCs and gas lines through each of the six capsules. In order to maintain gas tightness in each capsule, the original test train design had the thru tubes brazed at the top and bottom of each capsule. However, due to high axial and azimuthal thermal gradients in the thru tubes, high stresses would result with tube bowing and contact with the graphite sample holders. To alleviate the stresses and tube bowing, the current design has the thru tubes brazed only at the top of each capsule with the tube freed at the bottom. Neolube was applied around the tube at the bottom of the capsules to aid in assembly and act as a gasket. To further prevent capsule to capsule cross gas leakage, a nominal helium flow of 18 sccm at about 1 psig (6.9 $\mathrm{kPa}$-gauge) above the capsule pressure will be provided into the leadout cavity (and into the test train common plenum or void volume) via a mass flow controller. This small helium flow will provide an inward flow through the space between the capsule bottom heads and around the thru tubes. Experimental validations were conducted prior to start of irradiation to confirm the ingress gas flow and tube clearances are sufficient to prevent gas leakage from capsule to capsule.

\section{Power Shaping}

Three techniques are used to adjust the neutron flux incident upon the AGR-1 test articles to shape the temporal and spatial fuel power distribution. These techniques include the placement of a neutron absorbing shroud (hafnium) around the fuel, flowing a highly absorbing gas (helium-3) through the capsules, and placing a burnable absorber $\left(\mathrm{B}_{4} \mathrm{C}\right)$ near the fuel. 


\begin{tabular}{|l|lll|}
\hline \multirow{2}{*}{ AGR-1 Irradiation Experiment Test Plan } & Identifier: & \multicolumn{2}{l|}{ INL/EXT-05-00593 } \\
& Revision: & 3 & \\
& Effective Date: & $10 / 20 / 09$ & Page: 17 of 41 \\
\hline
\end{tabular}

Since compact fuel Stacks 1 and 3 directly face ATR's core center and shield Stack 2, fuel in Stacks 1 and 3 have higher neutron exposure and hence, higher rates of burnup and fluence. To achieve more uniform neutron exposure, a hafnium shroud is placed next to the inner wall of the capsules. The 0.25 $\mathrm{mm}(0.010 \mathrm{inch})$ thick Hf shroud extends $240^{\circ}$ around the circumference, centered between Stacks 1 and 3. The hafnium with a high neutron absorption cross section thus reduces the neutron exposure to Stacks 1 and 3 (in greater proportion than to Stack 2) resulting in lower neutron exposure gradients across the capsules. To maintain a uniform control gas gap, the remaining $120^{\circ}$ circumference is filled with 0.25 $\mathrm{mm}$ thick stainless steel. This configuration is illustrated in Figure 3-5.

Another power shaping technique used in AGR-1 is the addition of $\mathrm{B}_{4} \mathrm{C}$ to the graphite sample holders. Without this burnable absorber, fuel power, or heat generation rate, would be highest at beginning of life and drop exponentially as the fissile fuel content is consumed. For AGR-1 test fuel, this range of heat generation rates spans about $125 \mathrm{~W} / \mathrm{cm}^{3}$ from beginning of life to essentially, full burnup. Unfortunately, temperature control of the test fuel can only be maintained within a limited range of heat generation rates (about $50 \mathrm{~W} / \mathrm{cm}^{3}$ ) for a given control gas gap width and with a varying mixture of helium and neon sweep gas. To reduce the range of test fuel heat generation rates, $\mathrm{B}_{4} \mathrm{C}$ is added to the graphite sample holders. At beginning of life, the boron absorbs neutrons, reducing neutron exposure to the fuel and hence, reducing fuel power. As the irradiation continues, boron is depleted, neutron exposure to the fuel increases which increases fuel power. Eventually, with the boron consumed and the fissile fuel content diminished, fuel power decreases with continued irradiation. This temporal effect is shown in Figure 3-6.

Due to axial reductions in neutron flux at the top and bottom of the test train, reduced amounts of $\mathrm{B}_{4} \mathrm{C}$ will be required for the bottom and top capsules. Design calculations have determined that AGR-1 graphite sample holders will contain $7 \mathrm{wt} \% \mathrm{~B}_{4} \mathrm{C}$ in the middle capsules, Capsules 2 through 5 . The bottom capsule, Capsule 1, and the top capsule, Capsule 6, will each contain $5.5 \mathrm{wt} \% \mathrm{~B}_{4} \mathrm{C}$.

The control gas gap of each AGR-1 capsule is sized so that thermal control may be maintained during the peak in test fuel heat generation (depending on capsule, occurring at about 225 to 300 effective full power days [EFPD]) under normal reactor power. This allows for thermal control from the beginning of life through to approximately 500 EFPD. However, during a high power reactor cycle, or PALM cycle, reactor power is increased by as much as $43 \%$, which results in a corresponding increase in test fuel power. Should this occur during the time the fuel would be normally operating above about $90 \mathrm{~W} / \mathrm{cm}^{3}$ (between about 100 and 400 EFPD), the resulting increase in fuel power would exceed the power range that permits thermal control. To reduce fuel power during a PALM cycle, helium-3 will be used as the sweep gas. Helium-3 is a high neutron absorber that will significantly reduce test fuel power.

An analysis of the amount of power reduction resulting from the use of helium-3 sweep gas indicates that it is highly dependent upon the amount of porosity within the graphite sample holder and compacts that can contain the gas (Parry, 2006). For the worst case of a PALM cycle occurring at 250 EFPDs, the peak in test fuel heat generation, the peak fuel power increases from 133 to $191 \mathrm{~W} / \mathrm{cm}^{3}$ with the normally used helium-4 sweep gas. As illustrated in Figure 3-7, when helium-3 is used and 50\% of the porosity is filled, test fuel power is reduced to about $135 \mathrm{~W} / \mathrm{cm}^{3}$. Under these conditions, fuel remains under the targeted peak temperature. Should lesser amounts of the porosity be filled (deemed unlikely), the test fuel will likely exceed the targeted peak temperature. Measurements of available porosity within the graphite sample holders and compacts are planned for the next fiscal year at the INL. This information and precycle physics and thermal analyses will determine if thermal control can be maintained during a planned PALM cycle and if not, a program request will be made to reschedule the cycle. (Current ATR planning does not include a PALM cycle before July 2009. Therefore, AGR-1 could complete its irradiation without experiencing a PALM cycle.) 


\begin{tabular}{|l|lll|}
\hline \multirow{2}{*}{ AGR-1 Irradiation Experiment Test Plan } & Identifier: & INL/EXT-05-00593 \\
& Revision: & 3 & \\
& Effective Date: & $10 / 20 / 09$ & Page: 18 of 41 \\
\hline
\end{tabular}

\section{$\uparrow$ \\ Core Center}

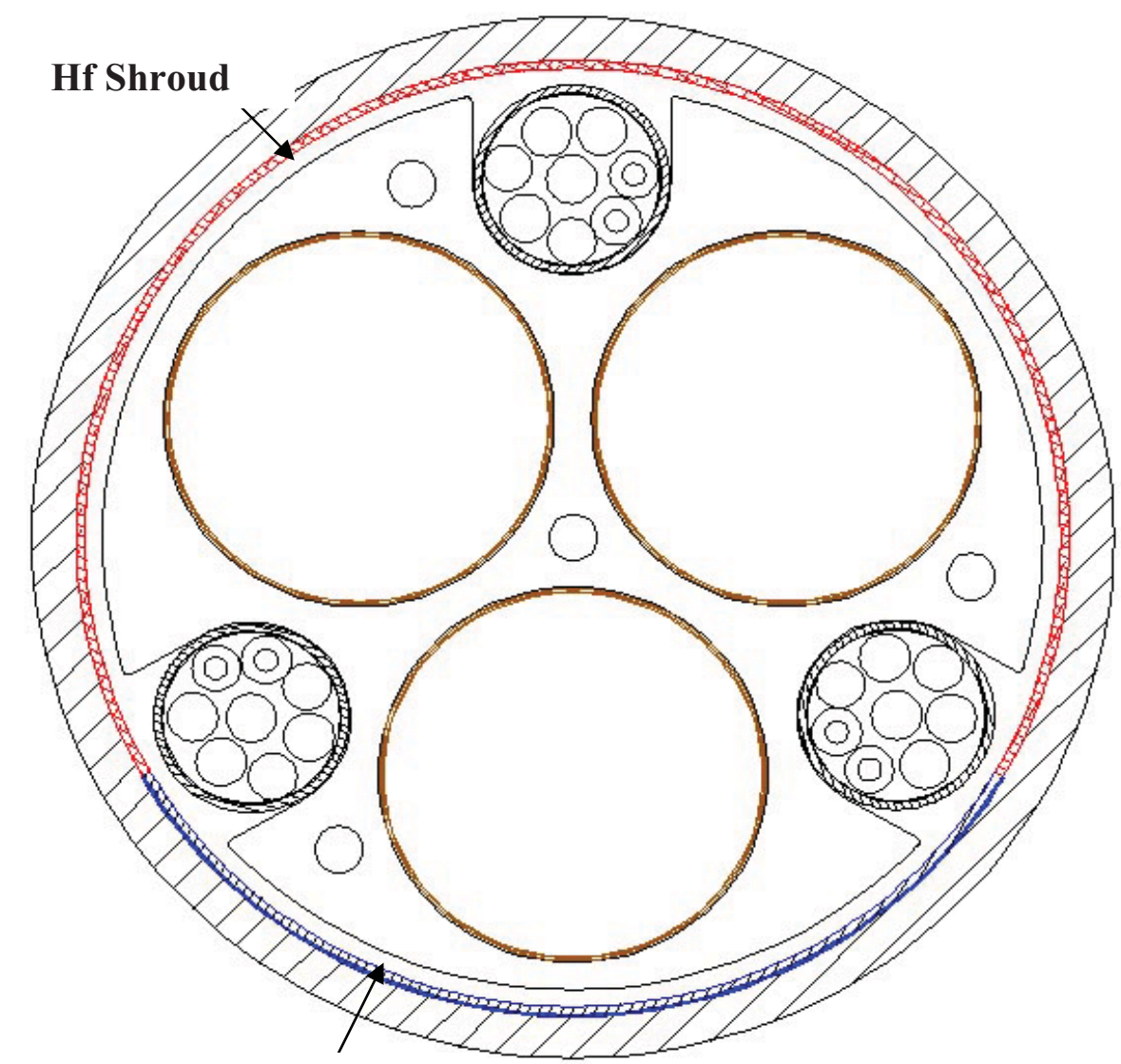

\section{Stainless Steel Shroud}

Figure 3-5. Placement of hafnium shroud in AGR-1. 
Form 412.09 (Rev. 10)

Idaho National Laboratory

\begin{tabular}{|l|lll|}
\hline \multirow{2}{*}{ AGR-1 Irradiation Experiment Test Plan } & Identifier: & INL/EXT-05-00593 \\
& Revision: & 3 & \\
& Effective Date: & $10 / 20 / 09$ & Page: 19 of 41 \\
\hline
\end{tabular}

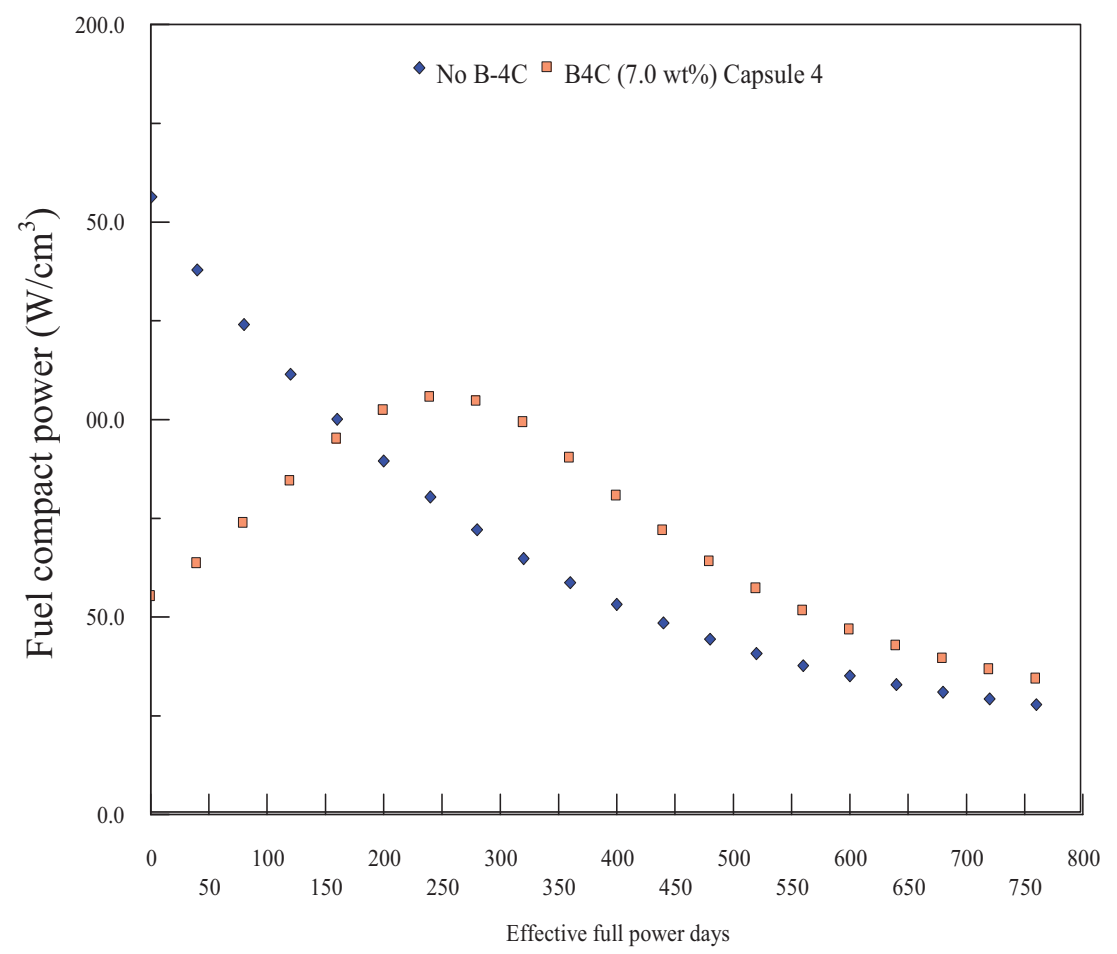

F 3-6. Effect of $\mathrm{B}_{4} \mathrm{C}$ on Effective Full Power Days

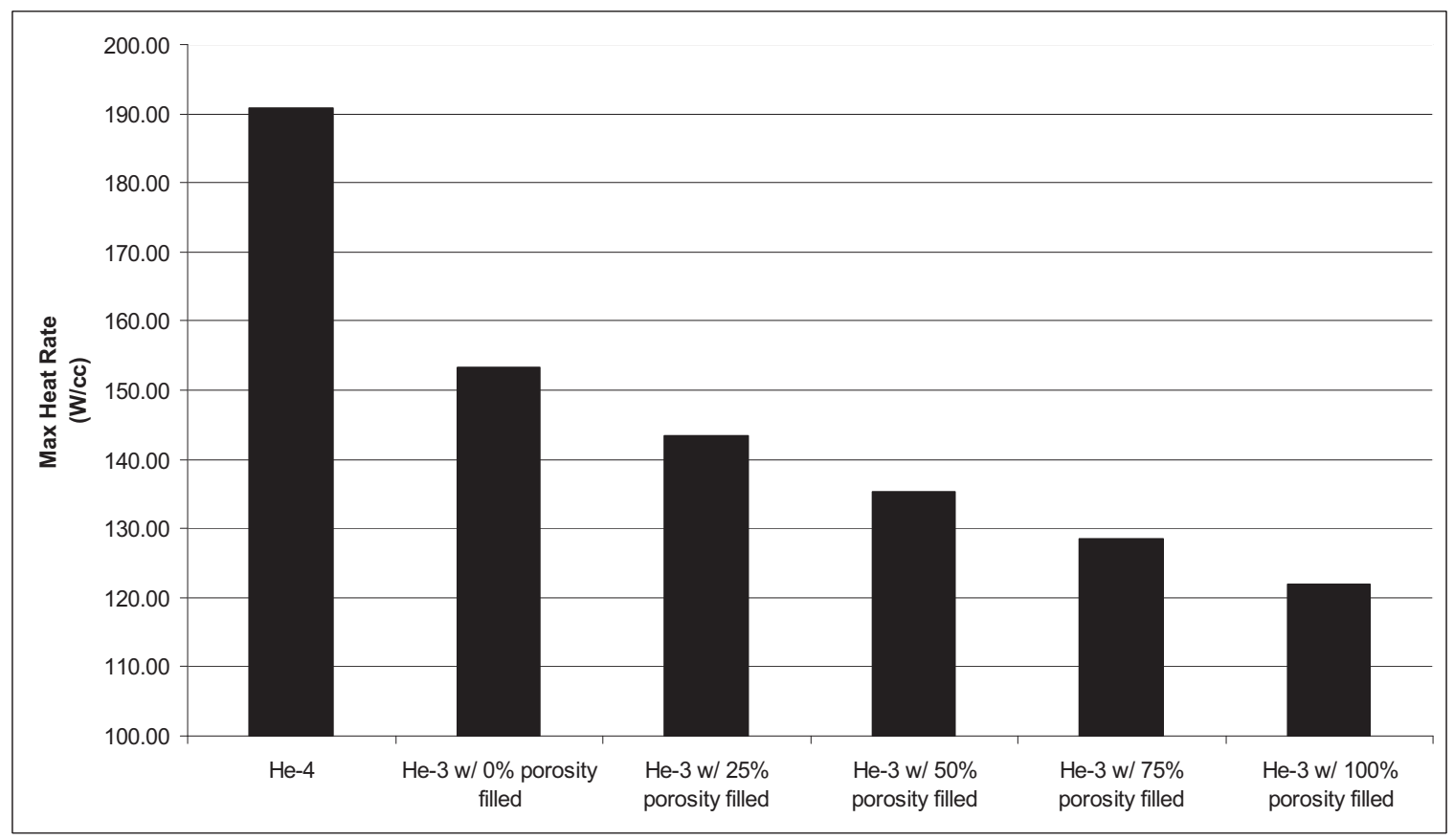

Figure 3-7. Effect of helium-3 sweep gas on fuel heat generation rate. 


\begin{tabular}{|l|lll|} 
AGR-1 Irradiation Experiment Test Plan & Identifier: & INL/EXT-05-00593 \\
& Revision: & 3 & \\
& Effective Date: & $10 / 20 / 09$ & Page: 20 of 41 \\
\hline
\end{tabular}

\subsection{Fission Product Monitoring System}

Each AGR-1 capsule will be continuously monitored for fission product gas release by the fission product monitoring system (FPMS). The FPMS consists of seven sets of gross radiation monitor and spectrometer detector pairs. One detector set is designated for each of the six capsules while the seventh detector set serves as a backup spare. A detector set is illustrated in Figure 3-8. In addition to the detectors, the FPMS has a manually operated grab sample for optional use.

Sweep gas carries released fission product gases from the capsules to the detector system under normal conditions with a transit time of about 150 seconds. The sweep gas enters the gross radiation monitor which uses a $\mathrm{NaI}(\mathrm{Tl})$ detector to detect each fuel particle failure up to the first 250 failures. Flow continues on to the spectrometer system which uses a HPGe (hyper pure germanium) detector. The spectrometer system measures radionuclide concentrations which are used to determine release to birth ratios. Under normal operation, computerized data acquisition, analysis and storage occurs continuously without operator intervention. 


\begin{tabular}{l|lll|}
\hline \multirow{2}{*}{ AGR-1 Irradiation Experiment Test Plan } & Identifier: & INL/EXT-05-00593 \\
& Revision: & 3 & Page: 21 of 41 \\
& Effective Date: & $10 / 20 / 09$ &
\end{tabular}

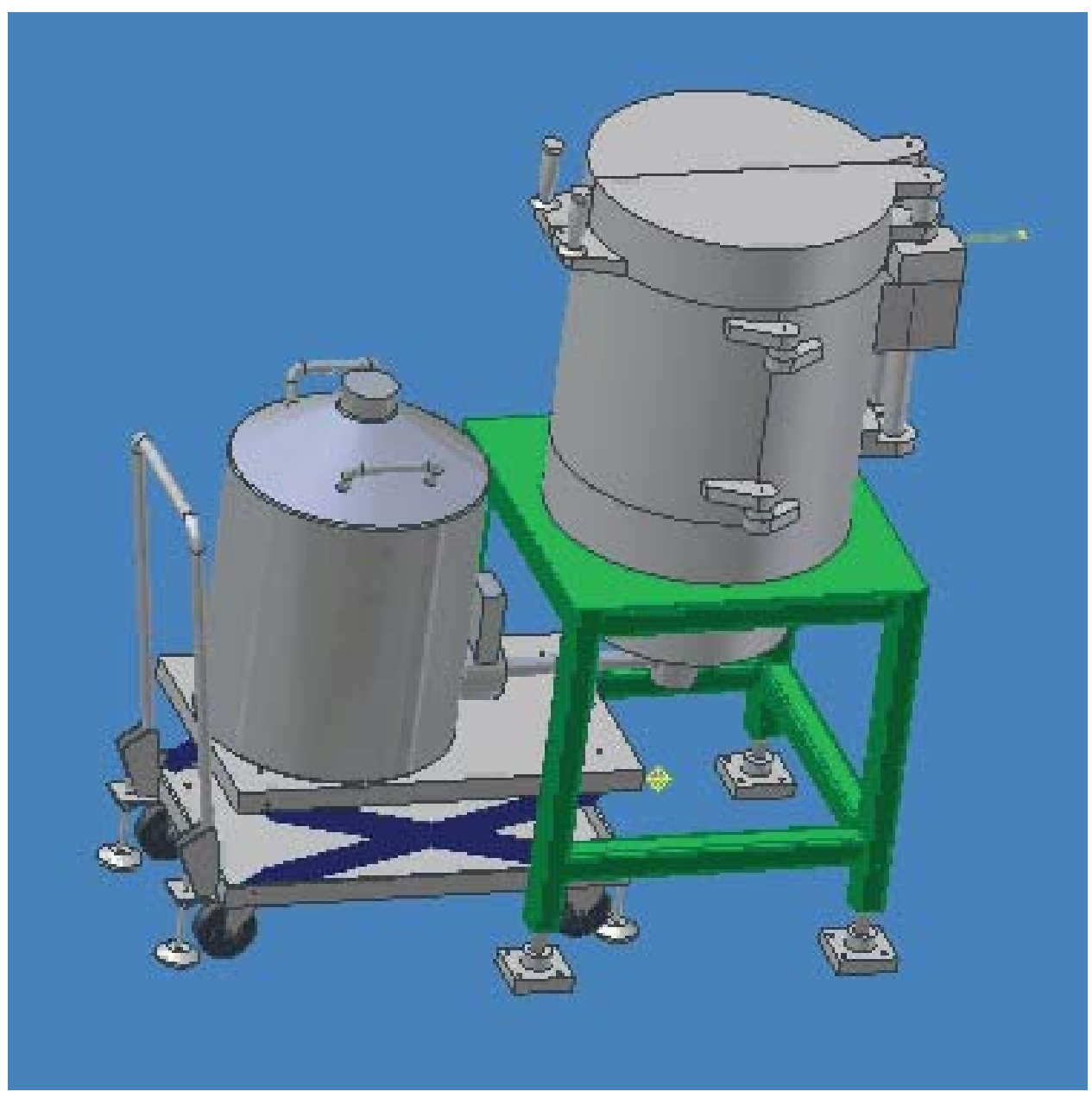

Figure 3-8. Gross radiation monitor and spectrometer detector for one AGR-1 sweep gas line. 


\begin{tabular}{|l|lll|}
\hline \multirow{2}{*}{ AGR-1 Irradiation Experiment Test Plan } & Identifier: & INL/EXT-05-00593 \\
& Revision: & 3 & \\
& Effective Date: & $10 / 20 / 09$ & Page: 22 of 41 \\
\hline
\end{tabular}

\section{TEST CONDITION REQUIREMENTS}

This section presents the irradiation conditions expected for the AGR-1 experiment. These calculated conditions, derived from the latest physics and thermal analyses, update the results presented in previous versions of this test plan.

\subsection{Particle Power}

Fuel power is restricted by specification (Maki, 2004) and by an operational need to control test temperature (which is defined as the ability to adjust and maintain fuel temperatures within a prescribed range). The instantaneous peak power per particle specification of $\leq 400 \mathrm{~mW} /$ particle is intended to limit peak kernel temperatures and temperature gradients across the particle which reduces fission product diffusion and potential fission product/SiC interactions. Temperature control is achieved by varying the composition of the sweep gas (between 100\% helium for high conductivity and 100\% neon for low conductivity) within the control gas gap surrounding the fuel. For a given gas gap width, this control can be maintained within a range, or window, of fuel heat generation rates.

In order to extend the time that thermal control can be maintained, boron carbide $\left(\mathrm{B}_{4} \mathrm{C}\right)$ is added to the graphite sample holders. This power shaping measure lowers the fuel heat generation rate early in life by absorbing neutrons and hence lowering the thermal neutron flux incident on the fuel. As the boron burns out later in life, the incident thermal neutron flux increases and fuel heat generation rates are dependant upon the remaining fissile fuel content. Since the AGR-1 test train extends axially beyond, both above and below, the flat portion of the ATR flux profile, the $\mathrm{B}_{4} \mathrm{C}$ content is reduced in the top and bottom capsule sample holders to compensate for the lower neutron flux.

Based on projected nominal ATR power cycles, the maximum and minimum compact average heat generation rates (Chang and Lillo, 2007) for AGR-1 are presented in Figure 4-1. A peak compact average power of $96 \mathrm{~mW} /$ particle is reached after about $250 \mathrm{EFPD}$. Considering that a conservative upper bound for compact peak-to-average power ratio is 1.1, the peak particle power is well within the specification limit of $\leq 400 \mathrm{~mW} /$ particle.

The effect of the boron carbide power shaping is clearly evident from Figure 4-1. Without $\mathrm{B}_{4} \mathrm{C}$, peak power would occur at beginning of life when the fuel fissile content is highest and the power would thereafter continuously decrease. With $\mathrm{B}_{4} \mathrm{C}$, the beginning of life power is reduced by about a factor of three (see Figure 3-6), and as the boron burns out, power increases to its peak after about 250 EFPD and then decreases. This power shaping thus extends duration of thermal controllability. 


\begin{tabular}{|l|lll|}
\hline \multirow{2}{*}{ AGR-1 Irradiation Experiment Test Plan } & Identifier: & INL/EXT-05-00593 \\
& Revision: & 3 & \\
& Effective Date: & $10 / 20 / 09$ & Page: 23 of 41 \\
\hline
\end{tabular}

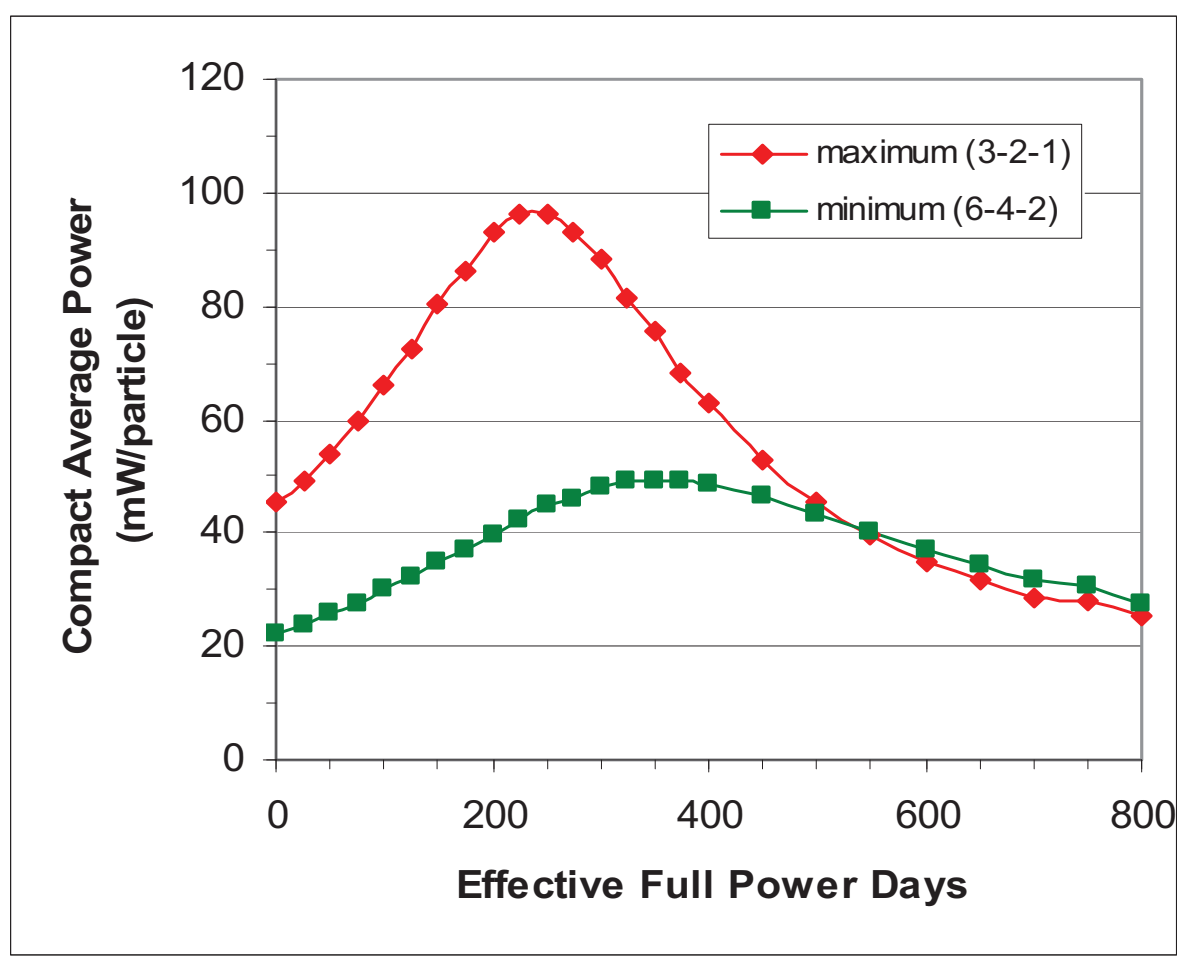

Figure 4-1. Compact average power for the maximum compact (Capsule 3 - Level 2 - Stack 1) and minimum compact (Capsule 6 - Level 4 - Stack 2).

\subsection{Temperature}

Three-dimensional, finite element, thermal calculations were performed at various irradiation times from beginning of life to 750 EFPD for the AGR-1 experiment (Ambrosek, 2007). These calculations were performed as described in Reference (Ambrosek, 2005) with the heat generation rates (Chang and Lillo, 2007) described above with optimized control gas gap widths and varying sweep gas compositions. Illustrative examples of these calculations are presented in Figure 4-2 which displays the thermal distribution of Capsule 3 fuel at three points in time.

The control gas gap width in each capsule was optimized, as stated above, such that the peak fuel temperature would be $1215 \pm 5^{\circ} \mathrm{C}$ during the peak in heat generation (occurring at 250 EFPD). Peak fuel temperatures have been lowered from $1250^{\circ} \mathrm{C}$ used in previous analyses. This reduction resulted from concerns pertaining to uncertainties in the thermal conductivity of the compact matrix material and graphite fuel bodies that could result in fuel being at higher temperatures for longer periods of time than desired. (Analyses use thermal conductivity correlations based on similar materials with compact matrix thermal conductivity correlations scaled by density owing to the significant reduction of AGR-1 compact matrix densities from the materials used to derive the correlations.) As this revision is being written, discussions among the Technical Coordination Team and program participants may result in peak AGR-1 fuel temperatures reverting back to near $1250^{\circ} \mathrm{C}$.

Maintaining peak fuel temperatures near $1215^{\circ} \mathrm{C}$ with appropriate sweep gas compositions, results in instantaneous peak temperature histories displayed in Figure 4-3. This analysis indicates that thermal 


\begin{tabular}{|l|lll|}
\hline \multirow{2}{*}{ AGR-1 Irradiation Experiment Test Plan } & Identifier: & \multicolumn{2}{l|}{ INL/EXT-05-00593 } \\
& Revision: & 3 & \\
& Effective Date: & $10 / 20 / 09$ & Page: 24 of 41 \\
\hline
\end{tabular}

control for each capsule can be maintained from up to 425 effective full power days for Capsule 3 to at least 750 effective full power days for Capsule 6. After these points, the capsules will be swept by $100 \%$ neon and fuel temperatures will continuously trend lower. The corresponding instantaneous, volume average temperature histories are displayed in Figure 4-4. Volume average temperatures initially decrease due to increasing amounts of helium used to maintain constant peak temperatures with increasing heat generation rates. After the peak in heat generation, increasing amounts of neon are used and the volume average temperatures increase until thermal control cannot be maintained (with $100 \%$ neon) and the volume average temperatures again decrease.

The instantaneous temperature histories were used to calculate the time-average peak temperatures, presented in Figure 4-5, and the time-average volume-average temperatures, presented in Figure 4-6. Time-average peak temperatures are well within the specification limit of $\leq 1250{ }^{\circ} \mathrm{C}$. However, five of the six capsules do not meet the time-average, volume-average temperature specification of $1150+30 /-75$ ${ }^{\circ} \mathrm{C}\left(1180-1075^{\circ} \mathrm{C}\right)$ for any significant irradiation duration. (It is noted that Capsule 1 does briefly enter within the specification range after 600 EFPD but then drops below after about 675 EFPD. Also, Capsule 6 drops slightly below the specification range between 400 and 675 EFPD.)

The reduction in peak fuel temperatures has had an expected result in lowering the achievable timeaverage, volume-average temperatures. This reduction was considered necessary due to the uncertainties involved in the thermal analysis and prompted the realization that the time-average, volume-average temperature specification, as originally written, may not be met. However, the achievable temperatures remain meaningful with time-average, volume-average temperatures ranging between 980 and $1080{ }^{\circ} \mathrm{C}$ after 725 EFPD.

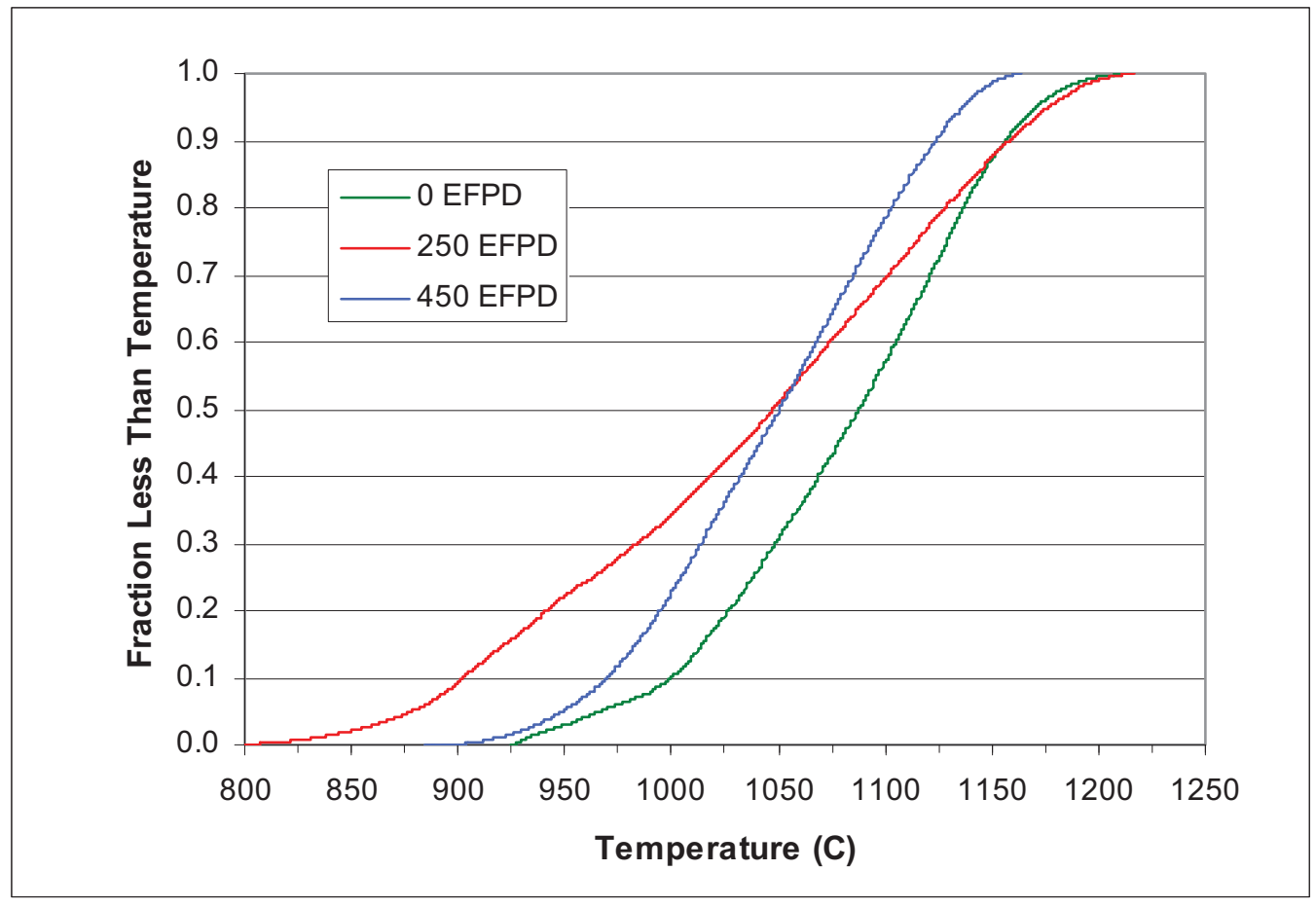

Figure 4-2. Thermal distributions in Capsule 3 fuel. 


\begin{tabular}{|l|lll|} 
AGR-1 Irradiation Experiment Test Plan & Identifier: & INL/EXT-05-00593 \\
& Revision: & 3 & Page: 25 of 41 \\
\hline
\end{tabular}

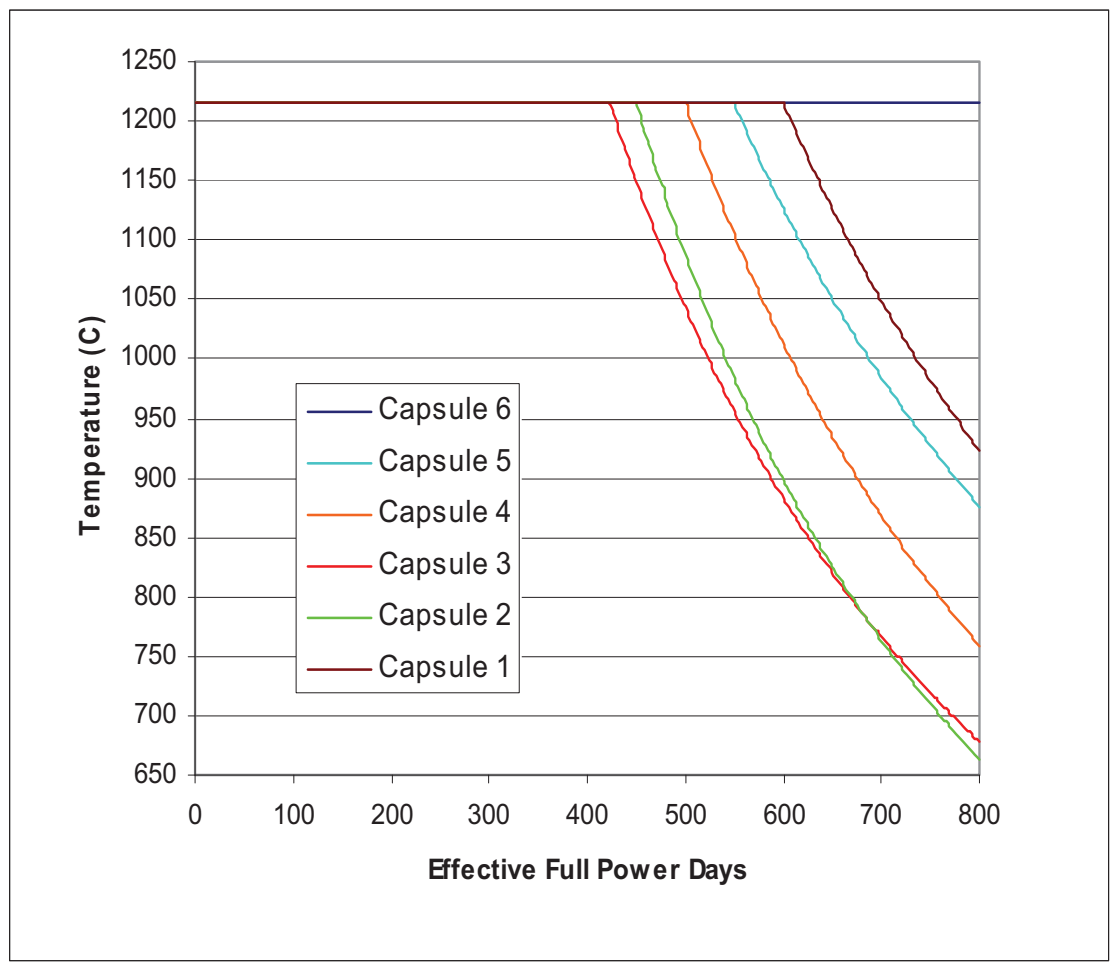

Figure 4-3. Instantaneous peak temperature as a function of effective full power days (EFPD).

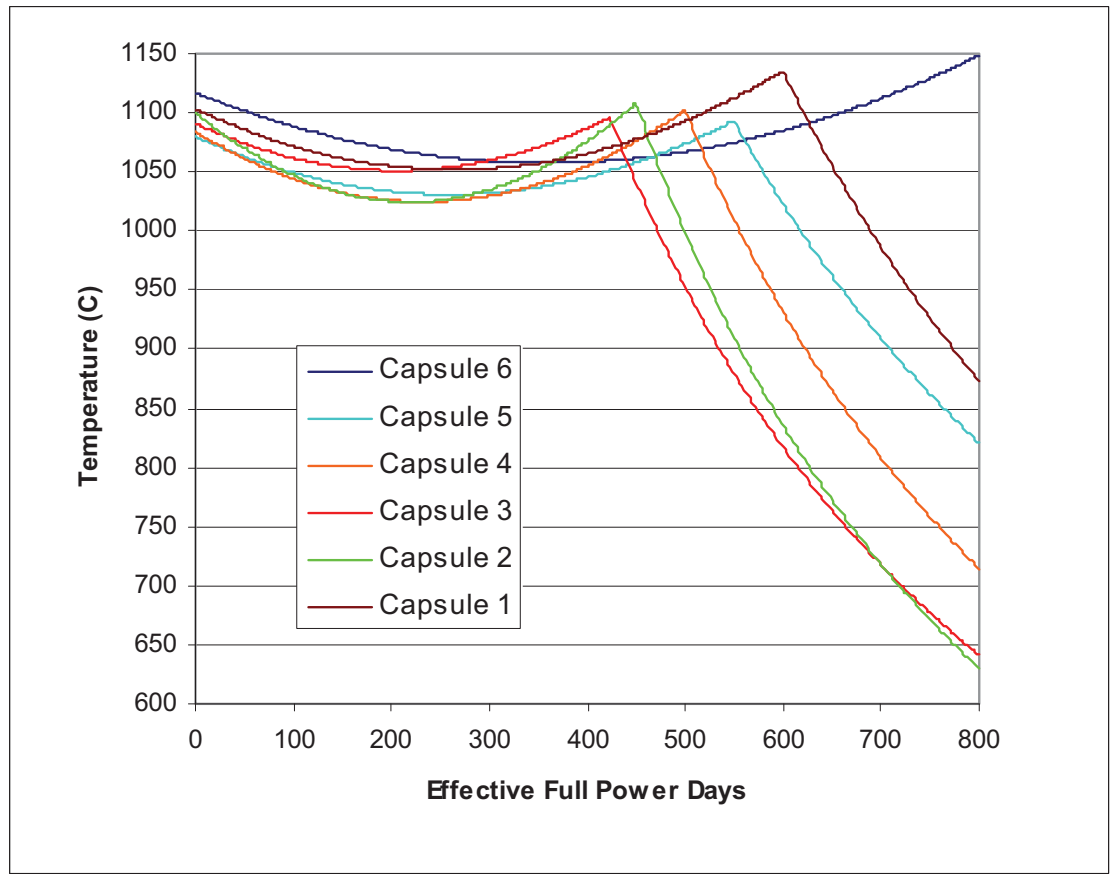

Figure 4-4. Instantaneous volume-average temperature as a function of effective full power days (EFPD). 


\begin{tabular}{|l|lll|} 
AGR-1 Irradiation Experiment Test Plan & Identifier: & INL/EXT-05-00593 \\
& Revision: & 3 & Page: 26 of 41 \\
\hline
\end{tabular}

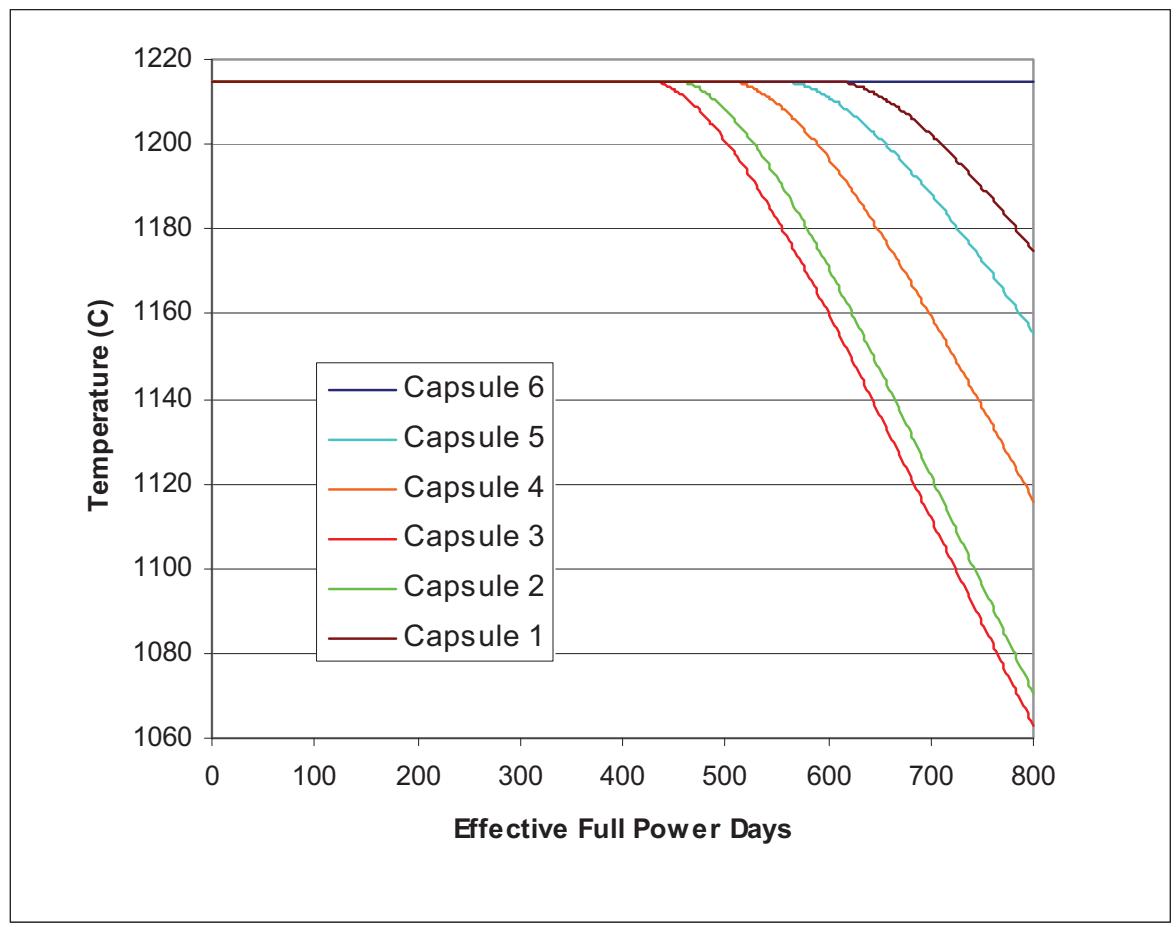

Figure 4-5. Time-average peak temperature as a function of EFPD (specification is $\leq 1250{ }^{\circ} \mathrm{C}$ ).

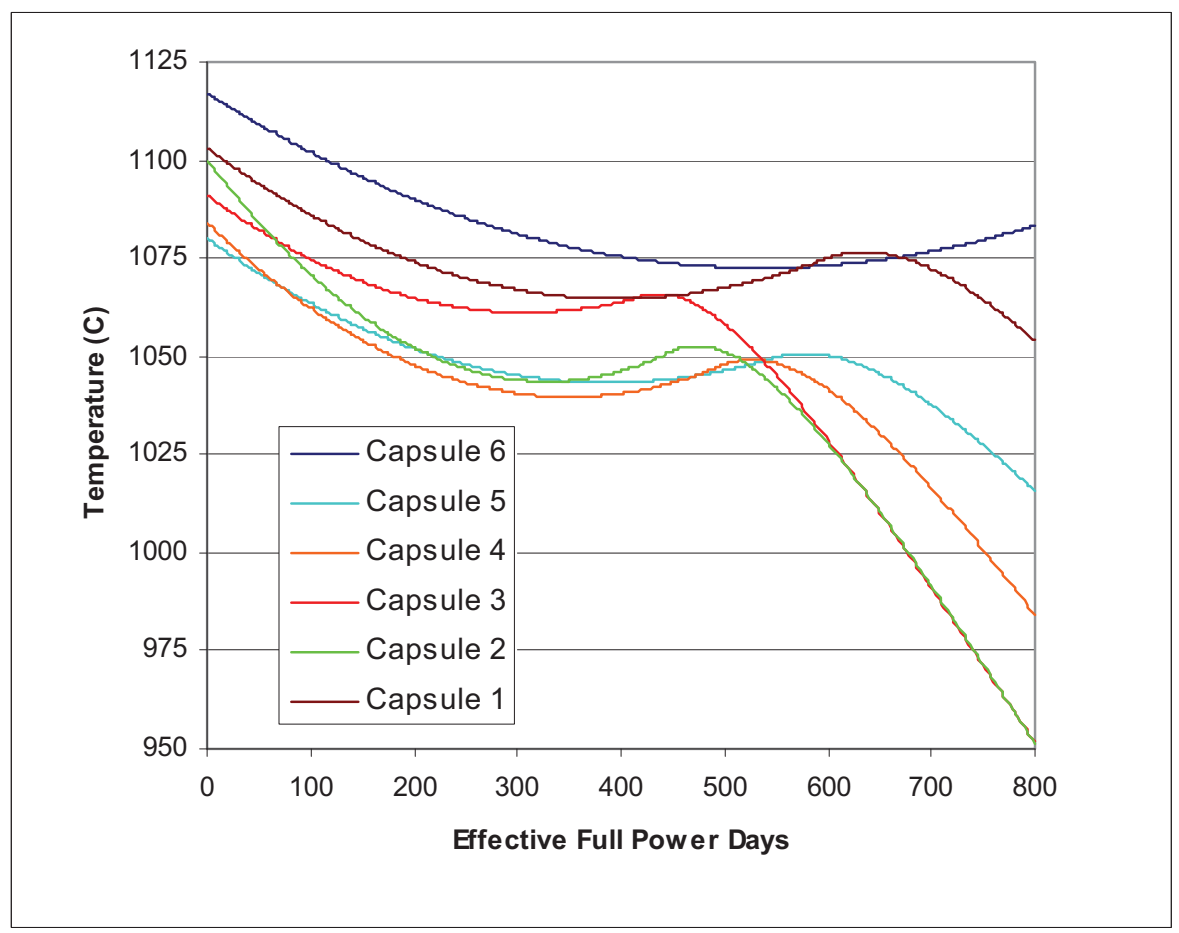

Figure 4-6. Time-average, volume-average temperature as a function of EFPD (specification is 1150 $\left.+30 /-75^{\circ} \mathrm{C}\right)$. 


\begin{tabular}{|l|lll|}
\hline \multirow{2}{*}{ AGR-1 Irradiation Experiment Test Plan } & Identifier: & INL/EXT-05-00593 \\
& Revision: & 3 & \\
& Effective Date: & $10 / 20 / 09$ & Page: 27 of 41 \\
\hline
\end{tabular}

\subsection{Fuel Burnup}

The intent of the test objectives and test specifications is for the fuel to obtain a "substantial" fraction of burnup within a reasonable amount of time (on the order of 2.5 calendar years). As such, the test specification lists a burnup goal, and not a requirement, for the majority of the fuel compacts to have a compact average burnup $>18 \%$ FIMA. However, a minimum compact average burnup is specified to be $>14 \%$ FIMA. This minimum burnup specification and the average burnup goal were established based on a preliminary analysis of an early test train conceptual design where most of the compacts would have a burnup between 19 and $21 \%$ FIMA and that the compacts at the extreme ends of the test train would exceed $14 \%$ FIMA.

Subsequent analyses incorporating current test train design changes (Chang and Lillo, 2007) indicate longer irradiation durations are required to achieve the same burnups as those used to establish the test specification. Some design changes such as inclusion of the $240^{\circ}$ hafnium shroud and varying the $\mathrm{B}_{4} \mathrm{C}$ content of the sample holders have had an effect on the time to reach essentially full burnup. Figure 4-7 presents the currently calculated capsule average burnups and Figure 4-8 displays the maximum and minimum compact average burnups. These results indicate that after approximately 725 EFPD of irradiation, the majority of the compacts will have reached the goal burnup of $18 \%$ FIMA and that perhaps two of the compacts will be under the minimum specified burnup of $14 \%$ FIMA (after 700 EFPD, 33 of the 72 compacts have burnups $\geq 18 \%$ FIMA, and three compacts have burnup $<14 \%$ FIMA; after 750 EFPD, 48 of the 72 compacts have burnups $\geq 18 \%$ FIMA, and one compact has burnup $<14 \%$ FIMA).

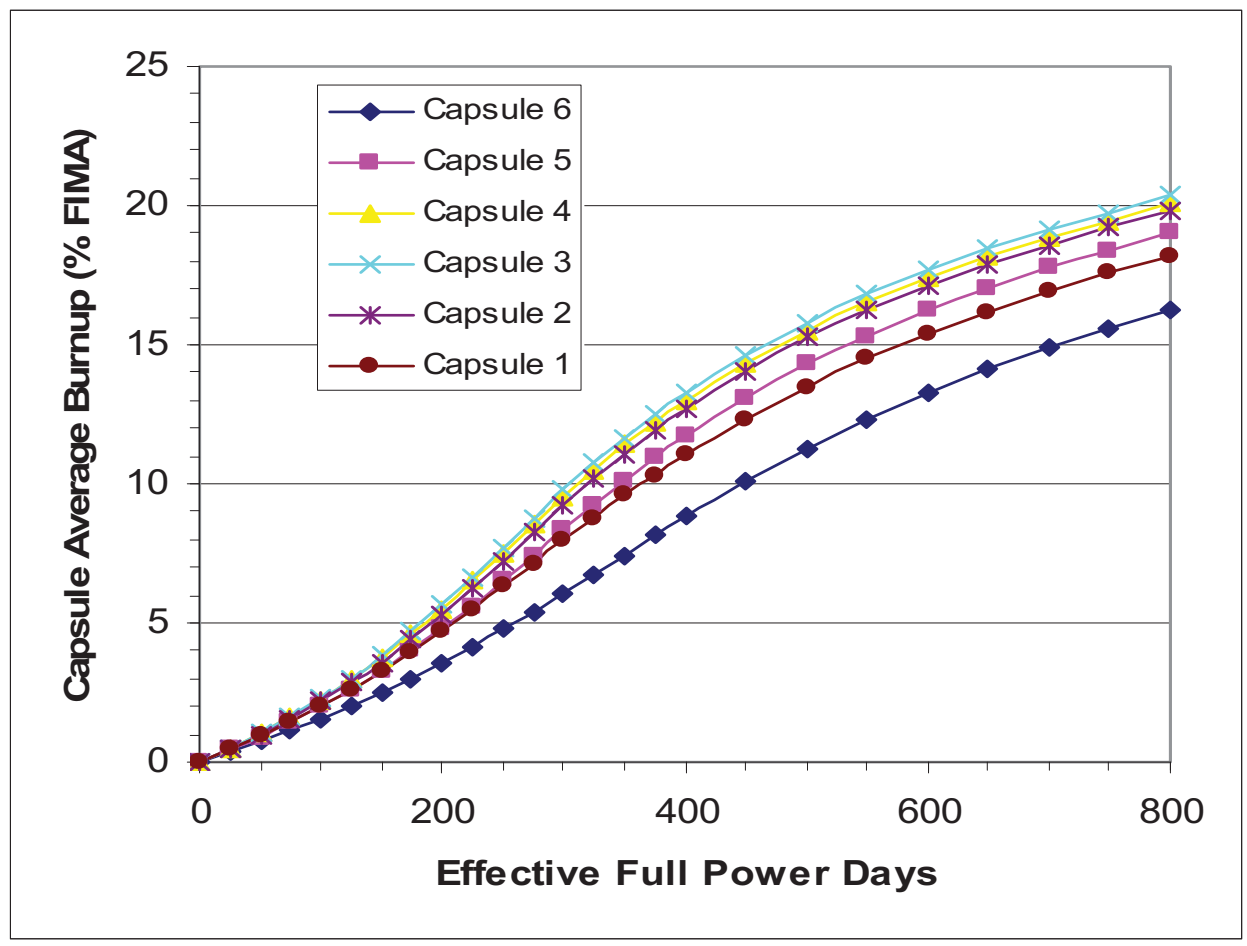

Figure 4-7. Capsule average burnups for AGR-1. 


\begin{tabular}{|l|lll|}
\multirow{2}{*}{ AGR-1 Irradiation Experiment Test Plan } & Identifier: & INL/EXT-05-00593 \\
& Revision: & 3 & Page: 28 of 41 \\
\hline
\end{tabular}

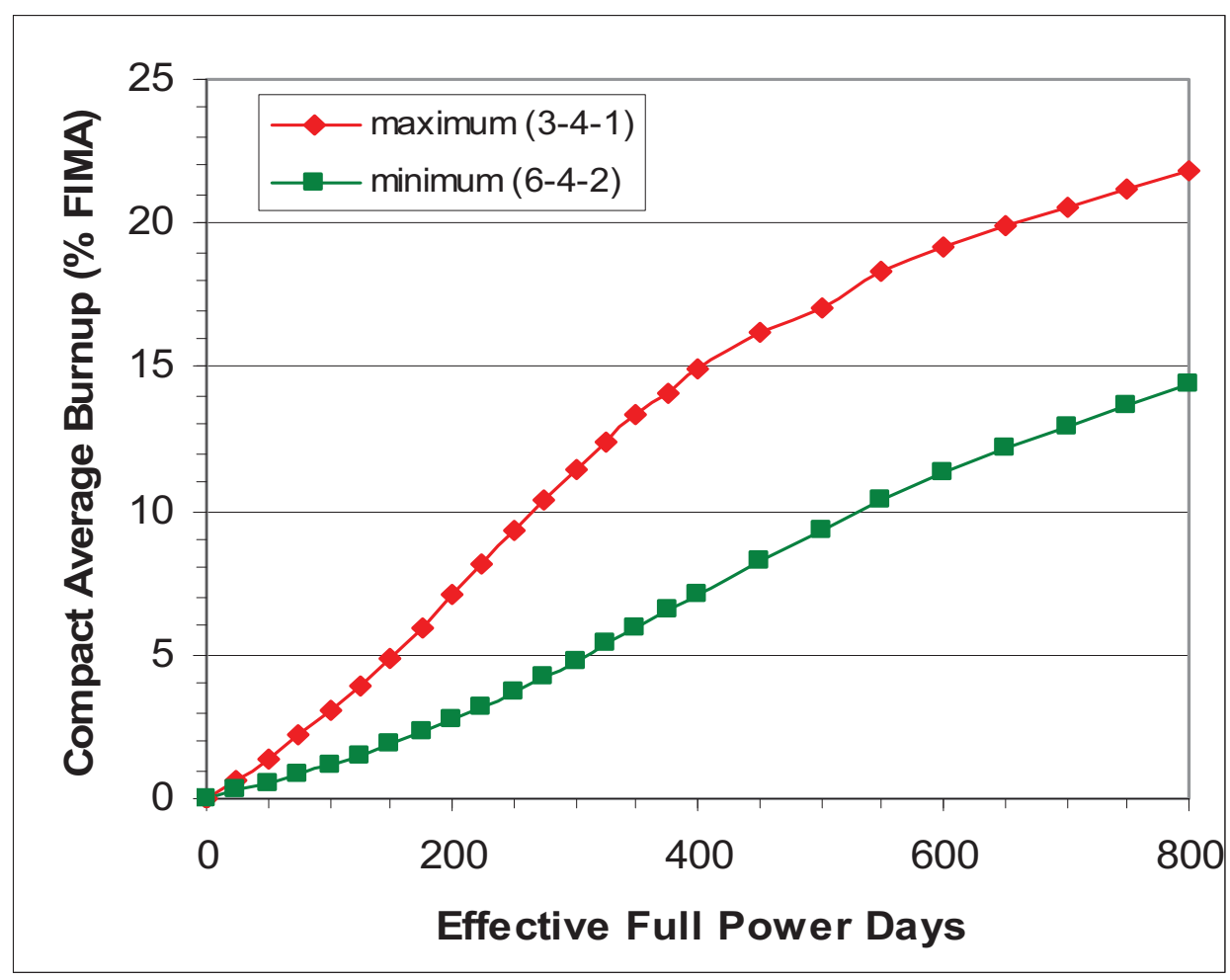

Figure 4-8. Compact average burnup for the maximum compact (Capsule 3 - Level 4 - Stack 1) and minimum compact (Capsule 6 - Level 4 - Stack 2).

\subsection{Fast Neutron Fluence}

The fast neutron fluence for each fuel compact is restricted by specification (Maki, 2004) to be $>1.5$ $\times 10^{25}$ and $<5 \times 10^{25} \mathrm{n} / \mathrm{m}^{2}$ for $\mathrm{E}>0.18 \mathrm{MeV}$. The upper limit is intended to bound expected VHTR service conditions while the lower limit is intended to ensure that the fuel pyrocarbon experiences the transition from creep-dominated strain to swelling-dominated strain.

Projections (Chang and Lillo, 2007) for capsule average fast neutron fluences are presented in Figure 4-9 and fluences for the maximum and minimum compacts are presented in Figure 4-10. The data indicate that the minimum specified fluence is reached for all compacts after about 400 EFPD and that the maximum specified fluence is exceeded after about 775 EFPD. 


\begin{tabular}{|l|lll|}
\hline \multirow{2}{*}{ AGR-1 Irradiation Experiment Test Plan } & Identifier: & INL/EXT-05-00593 \\
& Revision: & 3 & Page: 29 of 41 \\
\hline
\end{tabular}

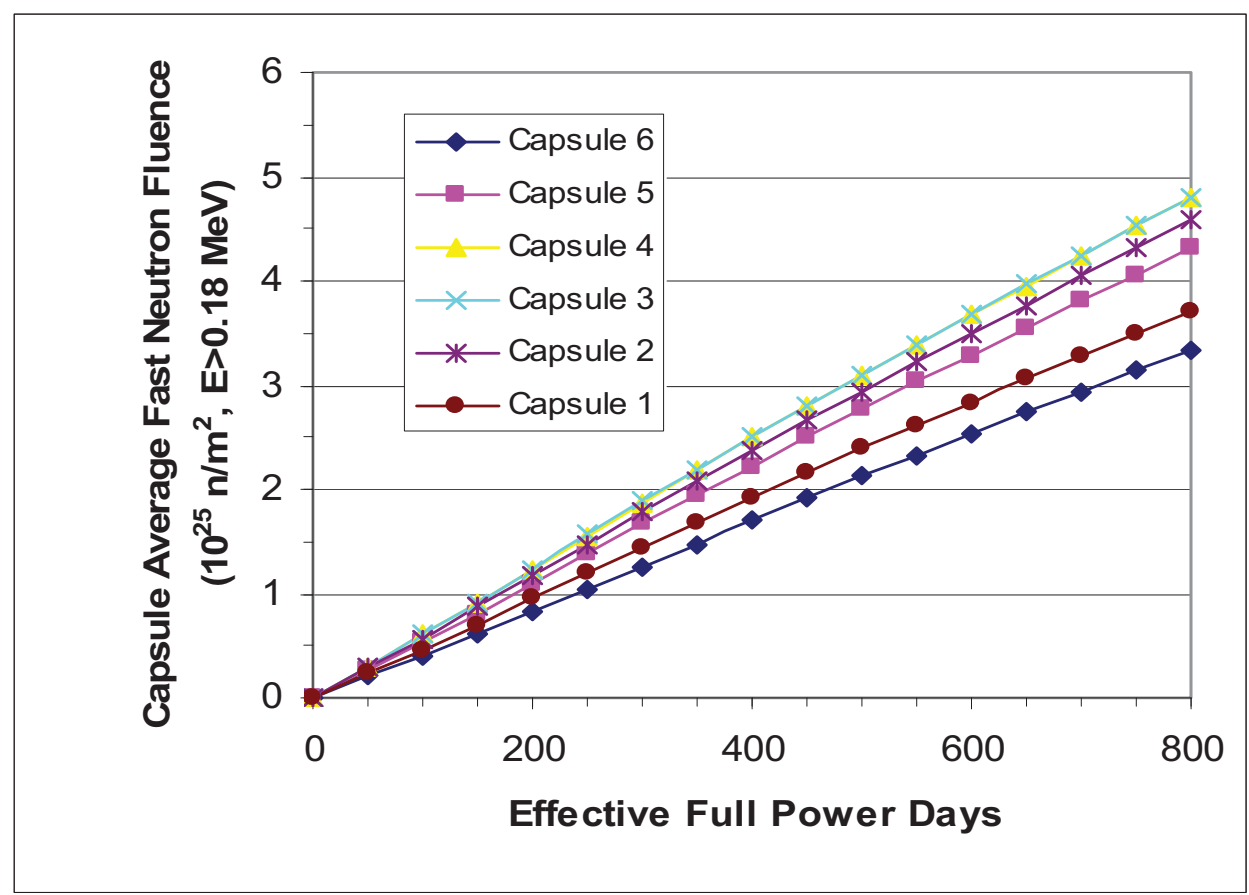

Figure 4-9. Capsule average fast neutron fluences for AGR-1.

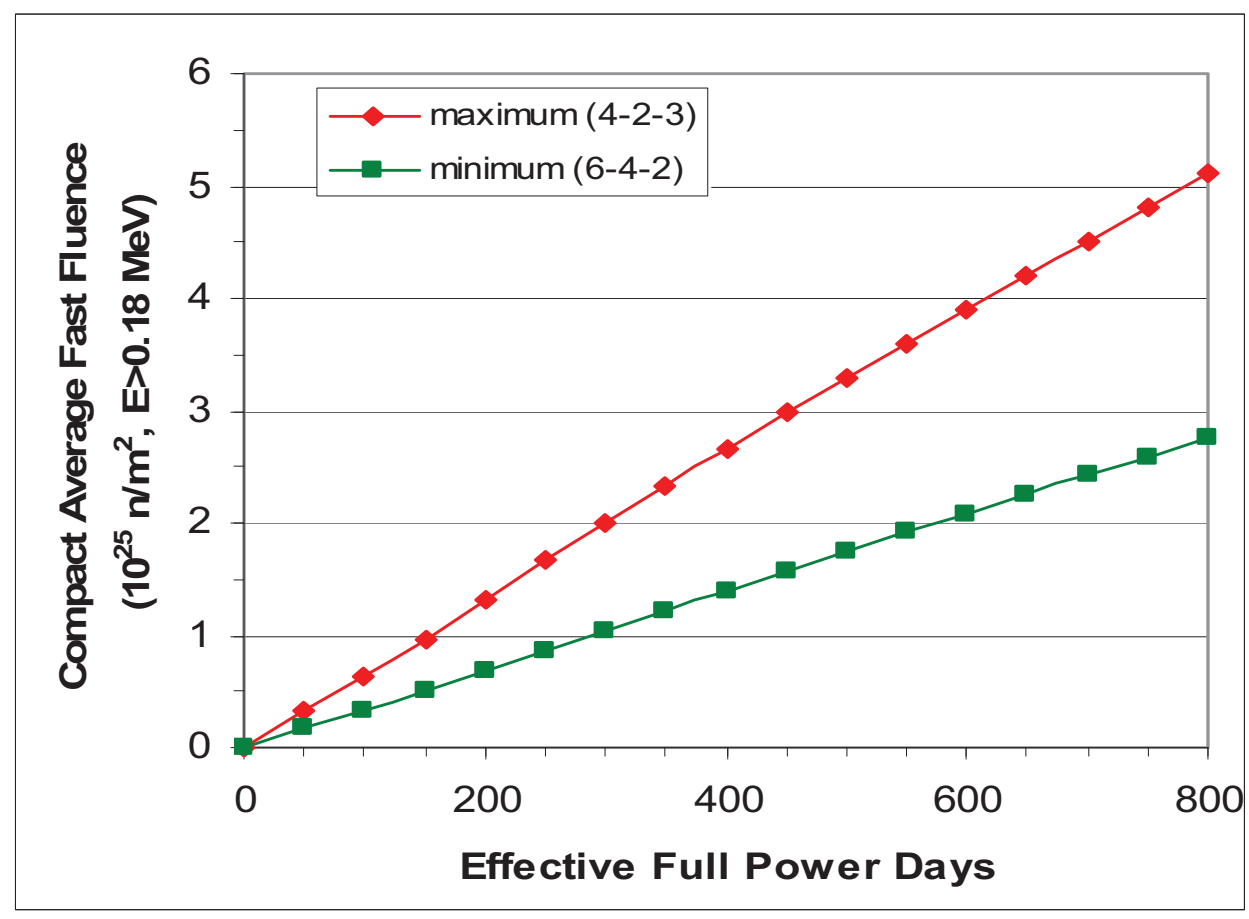

Figure 4-10. Compact average fast neutron fluence for the maximum compact (Capsule 4 - Level 2 Stack 3) and minimum compact (Capsule 6 - Level 4 - Stack 2). 


\begin{tabular}{|l|lll|}
\hline \multirow{2}{*}{ AGR-1 Irradiation Experiment Test Plan } & Identifier: & \multicolumn{2}{l|}{ INL/EXT-05-00593 } \\
& Revision: & 3 & \\
& Effective Date: & $10 / 20 / 09$ & Page: 30 of 41 \\
\hline
\end{tabular}

\subsection{Irradiation Duration}

Although not directly specified, the AGR-1 irradiation duration is constrained by the Technical Program Plan (Petti, 2005) assumption to limit the irradiation test acceleration to under two times that excepted in a real-time VHTR irradiation, and by the test specifications (Maki, 2004) for ancillary irradiation conditions. Since irradiating in a large B position in the ATR assures test acceleration is well under a factor of two, test duration is determined by evaluating the attributes of temperature, fast neutron fluence and burnup. This approach must balance increasing duration with decreasing temperatures and increasing burnup and fast fluence.

A summary of selected AGR-1 irradiation conditions and associated test specifications are presented in Table 4-1. As evident from the table, and discussed in Section 4.2, AGR-1 will not achieve specified time-average, volume-average temperatures during the test. Considering the uncertainties in these predicted temperatures and that the temperatures are nevertheless significant, the time-average, volumeaverage temperature specification may be relaxed for this evaluation. Also evident from Table 4-1, AGR1 will not achieve the minimum specified burnup levels within 2.6 calendar years. To increase burnup by extending the irradiation beyond $750 \mathrm{EFPD}$, results in higher fast neutron fluences that would violate test specification limits. Such an extension may also adversely impact the overall AGR program schedule.

Relaxing both time-average, volume-average temperature and minimum burnup requirements will still allow for the burnup goal (36 or more compacts with burnup $\geq 18 \%$ FIMA) and fast neutron fluence specification to be met with irradiation durations between 725 and 750 EFPD. Therefore, in order to maintain high as possible time-average, volume-average temperatures, a tentative target irradiation duration of 725 EFPD has been set. 


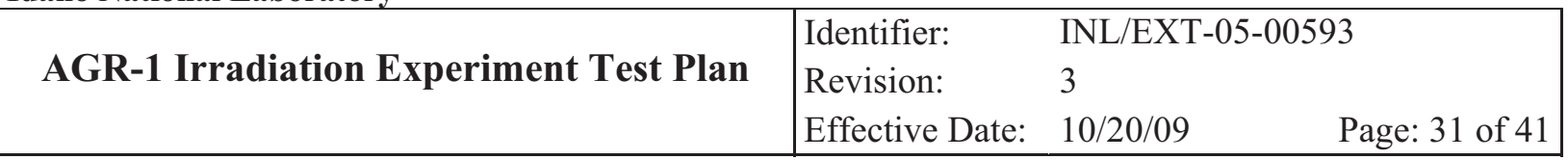

Table 4-1. Summary of AGR-1 irradiation conditions.

\begin{tabular}{|c|c|c|c|c|c|c|}
\hline \multirow[t]{2}{*}{ Parameter } & \multirow{2}{*}{$\begin{array}{l}\text { Test } \\
\text { Specification }\end{array}$} & \multicolumn{5}{|c|}{ Irradiation Duration $^{(\mathrm{a})}$ (EFPD) } \\
\hline & & 600 & 650 & 700 & 750 & 800 \\
\hline Calendar years ${ }^{(\mathrm{b})}$ & Not specified & 2.1 & 2.2 & 2.4 & 2.6 & 2.8 \\
\hline $\begin{array}{l}\text { Time-average peak } \\
\text { temperature range }{ }^{(\mathrm{c})}\left({ }^{\circ} \mathrm{C}\right)\end{array}$ & $\leq 1250$ & $\begin{array}{l}1161- \\
1215\end{array}$ & $\begin{array}{l}1137- \\
1215\end{array}$ & $\begin{array}{l}1112- \\
1215\end{array}$ & $\begin{array}{l}1088- \\
1215\end{array}$ & $\begin{array}{l}1063- \\
1215\end{array}$ \\
\hline $\begin{array}{l}\text { Time-average, volume- } \\
\text { average temperature range }{ }^{(\mathrm{c})} \\
\left({ }^{\circ} \mathrm{C}\right)\end{array}$ & $1075-1180$ & $\begin{array}{l}1028- \\
1075\end{array}$ & $\begin{array}{l}1011- \\
1076\end{array}$ & $\begin{array}{l}991- \\
1077\end{array}$ & $\begin{array}{l}972- \\
1080\end{array}$ & $\begin{array}{l}952- \\
1083\end{array}$ \\
\hline $\begin{array}{l}\text { Fast fluence range }{ }^{(\mathrm{d})} \\
\left(10^{25} \mathrm{n} / \mathrm{m}^{2}, \mathrm{E}>0.18 \mathrm{MeV}\right)\end{array}$ & $1.5-5.0$ & $\begin{array}{l}2.1- \\
3.9\end{array}$ & $\begin{array}{l}2.3- \\
4.2\end{array}$ & $\begin{array}{l}2.4- \\
4.5\end{array}$ & $\begin{array}{l}2.6- \\
4.8\end{array}$ & $\begin{array}{l}2.7 \\
5.1\end{array}$ \\
\hline $\begin{array}{l}\text { Number of compacts with } \\
\text { burnup }<14 \% \text { FIMA }\end{array}$ & 0 & 11 & 5 & 3 & 1 & 0 \\
\hline $\begin{array}{l}\text { Number of compacts with } \\
\text { burnup } \geq 18 \% \text { FIMA }\end{array}$ & Goal of $>36$ & 12 & 24 & 33 & 48 & 54 \\
\hline $\begin{array}{l}\text { Test train average burnup } \\
(\% \text { FIMA) }\end{array}$ & Not specified & 16.2 & 17.0 & 17.7 & 18.3 & 18.9 \\
\hline
\end{tabular}

Notes: (a) Shaded entries do not meet test specifications.

(b) Assumes 290 EFPD per calendar year.

(c) Range is on a per capsule basis.

(d) Range is on a per compact basis. 


\begin{tabular}{|l|lll|}
\hline \multirow{2}{*}{ AGR-1 Irradiation Experiment Test Plan } & Identifier: & \multicolumn{2}{|l|}{ INL/EXT-05-00593 } \\
& Revision: & 3 & \\
& Effective Date: & $10 / 20 / 09$ & Page: 32 of 41 \\
\hline
\end{tabular}

\section{MEASUREMENT REQUIREMENTS}

Several measurements are needed to demonstrate that the AGR-1 has reached the irradiation condition goals and test specifications. These conditions include time-average peak temperature, timeaverage volume-average temperature, fuel burnup, fast neutron fluence and fission gas release. Because the fuel compacts cannot be directly instrumented (which may induce particle failures), burnup, neutron fluence, and fuel temperature will be determined by calculations that require supporting measurement data. Each of these measurement categories are discussed below.

\subsection{Neutron Dosimetry}

Both thermal and fast neutron fluence measurements will be made for the AGR-1 experiment. The purpose of these measurements is to provide neutron exposure data that will support the calculations of the average burnup, fast neutron fluence and fission product inventory of each compact. This support may consist of a set of point values used to normalize physics calculations.

Following irradiation and test train disassembly, the encapsulated flux wires, described in Section 3.3, will be removed from each capsule. After removal from the encapsulation, the flux wires will be prepared and counted for their neutron induced radionuclide activities. Counting uncertainties will stay within specified limits of $\pm 10 \%$. Data collected from the neutron monitors will be corrected for decay according to standard procedures. Derived fast neutron fluence data will be further corrected to energies greater than $0.18 \mathrm{MeV}$. At all times, identification information (monitor type, serial number or similar code, original test train location) will remain with each neutron monitor.

\subsection{ATR Parameters}

ATR data that describes the core neutronic and thermal-hydraulic environment will be required. These data will be used to assist physics analysis (to calculate fuel burnup, heat generation rates and fast neutron fluences), assist thermal analysis (to calculate compact temperatures) and to support temperature control.

The ATR is a light water moderated, 93\% enriched uranium fueled test reactor. As shown in Figure $2-1$, the fueled core is arranged in a four-lobe clover leaf configuration. Each of the four corner lobes can be controlled at different powers to match the requirements of various in-pile experiments. ATR is rated at a total thermal power of $250 \mathrm{MW}$, however, the reactor is normally operated in the range of 105 to 115 MW to meet most experiment needs.

ATR data that will be provided include individual lobe powers, shim cylinder positions, and core inlet temperatures. These data will be recorded, and backed up on a separate storage device, once every minute. 


\begin{tabular}{|l|lll|}
\hline \multirow{2}{*}{ AGR-1 Irradiation Experiment Test Plan } & Identifier: & \multicolumn{2}{l|}{ INL/EXT-05-00593 } \\
& Revision: & 3 & \\
& Effective Date: & $10 / 20 / 09$ & Page: 33 of 41 \\
\hline
\end{tabular}

\subsection{Temperature Measurements}

Temperature measurements will be performed by TCs terminating within the graphite sample holders of each capsule. These measurements will be used to support thermal analyses of the test train which ultimately will determine fuel temperatures. TC measurements will also be used to support temperature control of the experiment. For this function, one TC per capsule is designated as the control TC. Measurements from the control TCs provide feedback to the automated sweep gas control system which adjusts gas blend to maintain reference temperatures.

AGR-1 TCs will have at least an-installed accuracy of $\pm 2 \%$ of reading as required by the test specification. During normal and abnormal operation, TC data will be recorded, and backed up on a separate storage device, once every minute.

\subsection{Sweep Gas Parameters}

In addition to the TC measurements, several sweep gas parameters will be required for thermal analyses and temperature control. These include pressure, mass flow rates of each constituent gas, and moisture content. Sweep gas pressures and constituent mass flow rates (which determine gas mixture ratios) will be used in physics and thermal analyses of the test train. Moisture content measurements (measured on the outlet side of the capsule and compared to the gas supply verification measurement) will provide indicators of capsule integrity.

Capsule inlet pressure will be measured to within $\pm 0.007 \mathrm{MPa}$ ( \pm 1 psia) with constituent mass flow rates measured within 1\% RMS (root mean square). Moisture data will be converted to ppm-vol (parts per million by volume) relative to $15 \mathrm{psi}$. These data will be recorded, and backed up on a separate storage device, once every minute.

\subsection{Fission Gas Release Monitoring}

Fission gas release measurements will provide indicators of fuel irradiation performance. Gross radiation monitors will continuously measure the sweep gas from each capsule to indicate fuel particle failures. Spectrometer detectors will measure radionuclide concentrations to determine release to birth rate $(\mathrm{R} / \mathrm{B})$ ratios of selected nuclides. $\mathrm{R} / \mathrm{B}$ values provide indicators of initial fuel quality and also provide indications of fuel failure.

The gross radiation monitors will have sufficient sensitivity to detect every fuel particle failure, up to and including the first 250 failures, from each capsule. These fuel particle failures are indicated by a rapid rise and drop, or "spike", in the measured count rate. Such spikes are a result of a sudden release of stored fission product inventory. Measured spectra will be automatically stored and backed up.

The spectrometer detector systems will measure the concentrations of various krypton and xenon isotopes in the sweep gas from each capsule. During normal operation, ten hour counting intervals will measure the concentrations of Kr-85m, Kr-87, Kr-88, Kr-89, Kr-90, Xe-131m, Xe-133, Xe-135, Xe$135 \mathrm{~m}, \mathrm{Xe}-137, \mathrm{Xe}-138$, and Xe-139. These concentrations will be converted to fuel release rates, and with calculated birth rates, $\mathrm{R} / \mathrm{B}$ ratios will be determined. Measured spectra will be automatically stored and backed up. 


\begin{tabular}{|l|lll|}
\hline \multirow{2}{*}{ AGR-1 Irradiation Experiment Test Plan } & Identifier: & INL/EXT-05-00593 \\
& Revision: & 3 & \\
& Effective Date: & $10 / 20 / 09$ & Page: 34 of 41 \\
\hline
\end{tabular}

During reactor outages, the capsules will be swept with pure helium-4. This sweep gas will be analyzed for Xe-133 and Xe-135. These xenon concentrations will be used to calculate R/Bs of their parent iodine isotopes. Presence of the fission product iodine is also an indicator of fuel performance.

\subsection{Data Validation and Qualification}

Measured data will be evaluated for validation and then qualified for use. It is currently planned that a commercial software package will process the data for this purpose. The following parameters will be evaluated: TC temperatures, constituent sweep gas flow rates and pressure, fission product monitor system count rates and spectra, and neutron monitor analyses. 


\begin{tabular}{|l|lll|}
\hline \multirow{2}{*}{ AGR-1 Irradiation Experiment Test Plan } & Identifier: & INL/EXT-05-00593 \\
& Revision: & 3 & \\
& Effective Date: & $10 / 20 / 09$ & Page: 35 of 41 \\
\hline
\end{tabular}

\section{OPERATIONAL REQUIREMENTS}

All operational activities associated with the AGR-1 experiment shall comply with all applicable INL and ATR standard procedures. These activities will also comply with all safety and quality assurance requirements outlined in Section 7. Activities requiring special or unique consideration are identified below.

\subsection{Pre-irradiation}

Prior to final test train assembly, confirmatory physics and thermal analyses will be made using actual fuel characterization data, expected ATR operating conditions, and material properties for the borated graphite sample holders. Based upon these results, the control gas gap width may be re-optimized. Also, stress analysis of the test train may be re-evaluated.

Following receipt and inspection of the AGR-1 fuel compacts, baseline and fuel variant compacts will be selected for irradiation based upon integrity and dimensions. The sample holders in each capsule will be specifically bored to accept these compacts based upon compact diameters.

After assembly, test train and fission product monitor components and sub-systems will undergo inspection, testing and calibration as-needed. This will include, but is not limited to, leak testing of all pressure boundaries and gas lines, and continuity check for all TCs. Following these activities, a review will be conducted whereby any findings will be corrected.

Following successful completion of the review and obtaining all appropriate ATR approvals, the AGR-1 test train will be inserted into the B-10 position of the ATR. Air within the lead and gas lines will be purged and final component inspections will be performed.

\subsection{Irradiation}

During irradiation, temperature control is automatically maintained by the gas control system. This system requires temperature feedback from a control TC within each capsule. Should a control TC fail, a previously selected back-up TC within the same capsule will then be used as the control TC and the reference control temperature reset based on thermal analysis calculations. Should all TCs fail within a capsule, results from physics and thermal analyses supported by the operating history of an adjacent capsule will be used to manually set the gas blends of the affected capsule. Ultimately, should all TCs fail within the test, temperature control may be based upon predictive thermal analyses, augmented by analyses of fission product gas release which is sensitive to temperature.

Thermocouple drift will be monitored by analyses. With actual gas mixes and predicted heat generation rates from physics analyses, the thermal model will be adjusted and calibrated to TC readings during the start of the first irradiation cycle (about two days after reactor start-up so that xeon equilibrium is reached). Thereafter, thermal model results will be compared to the TC readings. Should the difference between model predictions and actual readings of a control TC differ by more than $50^{\circ} \mathrm{C}$ (about 4 to $5 \%$ of reading), control set points for the gas mix system will be adjusted to compensate for the TC drift.

Current ATR planning does not include a PALM cycle before July 2009. Should this planning change, physics and thermal analyses will evaluate the effects of scheduled PALM cycles. These analyses will determine if standard sweep gas will maintain thermal controllability or the use of helium-3 sweep gas will allow for continued irradiation. In the unlikely event that these analyses indicate 


\begin{tabular}{|l|lll|}
\hline \multirow{2}{*}{ AGR-1 Irradiation Experiment Test Plan } & Identifier: & \multicolumn{2}{|l|}{ INL/EXT-05-00593 } \\
& Revision: & 3 & \\
& Effective Date: & $10 / 20 / 09$ & Page: 36 of 41 \\
\hline
\end{tabular}

temperature control cannot be maintained and targeted peak fuel temperatures will be exceeded, even with helium-3, the program may request that the PALM cycle be rescheduled.

During any switch in control gas (to pure helium-4, to helium-3, to a neon helium mix, etc.), flows of the common plenum gas and each control gas will be appropriately adjusted to ensure that continuity is maintained in the pressure differential between the common plenum and each capsule. This ensures that cross flow between capsules is avoided. After each ATR shutdown and during the entire outage, the control gas will be switched to pure helium- 4 for each capsule, and the helium will continuously flow through each capsule at the nominal operation flow rate. The plenum flow will also be maintained at its nominal operational flow rate.

Should a capsule experience excessive fuel particle failures, on the order of $5 \%$ or approximately 2500 particles, sweep gas to the capsule will be set to consist of $100 \%$ helium- 4 . The helium sweep gas will be maintained at the nominal operation flow rate until the end of the irradiation.

Indicators of moisture ingress (sweep gas outlet moisture content higher than inlet content) will be closely monitored. Past experience has shown that once the presence of moisture is detected, the content rapidly increases. Should a rapid increase in moisture be observed in a capsule, the test train may be removed from the reactor at the next scheduled reactor outage to avoid significant water-graphite interactions possibly compromising other capsules via gaps that may form (due to reactions between steam and Neolube) around the thru tubes.

Program participants may be able to view time-series data on-line via a secured site. Viewable data should include at least TC measurements, sweep gas parameters, and gross radiation monitor count rates. Content and format for this possible data presentation has not been fully defined.

As a result of cycle to cycle variations in ATR lobe powers, accumulated burnup and fast neutron fluence for the AGR-1 test articles must be periodically updated based on as-run data. These as-run data reports will be issued at least after $50 \%$ and after $75 \%$ of projected test completion, and then after the end of each reactor cycle to the test completion. In addition to fuel burnups and fast neutron fluences, the asrun data reports will present fuel temperature averages, R/B values and estimated number of fuel particle failures.

\subsection{Post-irradiation}

The AGR-1 test train will be removed from the reactor after completion of the irradiation. For removal, the TCs and gas lines will be disconnected at the reactor vessel penetration flange (where the leadout passes through the reactor wall). The gas lines will then be capped and a cover installed on the test train leadout flange. The entire test train is then lifted from the B-10 test position and passed through the transfer chute to the ATR canal.

After completion of the irradiation, the test train will cool in the canal for about two months before being transferred to a hot cell for disassembly. Preliminary PIE will be conducted during and immediately after disassembly. Plans for follow-on detailed PIE have not yet been finalized.

A Quick-look Irradiation Test Results report will be issued within six weeks of the completion of the irradiation. Within nine months of test completion, a Final Irradiation Test Results report will be issued. Results from PIE and safety testing will be documented separately after the completion of those activities. 


\begin{tabular}{|l|lll|}
\hline \multirow{2}{*}{ AGR-1 Irradiation Experiment Test Plan } & Identifier: & \multicolumn{2}{l|}{ INL/EXT-05-00593 } \\
& Revision: & 3 & \\
& Effective Date: & $10 / 20 / 09$ & Page: 37 of 41 \\
\hline
\end{tabular}

\section{SAFETY AND QUALITY ASSURANCE \\ 7.1 Safety}

The design, fabrication, installation, operation and disassembly activities of the AGR-1 experiment shall comply with all applicable health, safety, and environmental requirements. These activities and their corresponding requirement directives are listed in Table 7-1.

Table 7-1. AGR-1 safety requirements.

\begin{tabular}{|l|l|}
\hline Activity / Component & Requirements \\
\hline Design, installation and operation of test lead & $\begin{array}{l}\text { ATR Technical Safety Requirements } \\
\text { Upgraded Final Safety Analysis Report }\end{array}$ \\
\hline Capsule containment tube & ASME Boiler and Pressure Vessel Code \\
\hline Mechanical design & Applicable sections of ASME and AWS Codes \\
\hline Nuclear materials accountability & Applicable DOE orders \\
\hline Radioactive material shipments & Applicable DOE orders \\
\hline
\end{tabular}

\subsection{Quality Assurance}

Quality assurance activities associated with the AGR-1 experiment shall comply with all applicable requirements set forth in:

- INL Quality Assurance Program based on ASME NQA-1 2000

- $\quad$ AGR Quality Program Plan, PLN-1468

- Reactor Technology Complex (INL) Site Specific QA Implementation Procedures and Forms.

Activities requiring specific quality requirements include, but are not limited to the following:

- Capsule design review

- Capsule fabrication

- Component and system operational testing

- Test calibration

- Operational procedures

- Computer software control 


\begin{tabular}{|l|lll|} 
AGR-1 Irradiation Experiment Test Plan & Identifier: & INL/EXT-05-00593 \\
& Revision: & 3 & \\
& Effective Date: & $10 / 20 / 09$ & Page: 38 of 41 \\
\hline
\end{tabular}

- Irradiation data collection

- Neutron monitor analysis

- Fission product gas analysis

- Data management

- Data validation

- Reporting. 


\begin{tabular}{|l|lll|}
\hline \multirow{2}{*}{ AGR-1 Irradiation Experiment Test Plan } & Identifier: & \multicolumn{2}{l|}{ INL/EXT-05-00593 } \\
& Revision: & 3 & \\
& Effective Date: & $10 / 20 / 09$ & Page: 39 of 41 \\
\hline
\end{tabular}

\section{PROGRAM CONSTRAINTS AND SCHEDULE}

Several possible programmatic constraints may affect the scheduling and accomplishment of significant activities presented in this test plan. Some of these constraints are discussed below.

The AGR-1 test train was ready to insert into ATR on September 30, 2006. Irradiation started on December 23, 2006.

An irradiation duration of approximately 2.5 calendar years is currently envisioned. This duration may be shortened due to significant test train or fuel failures or lengthened to gain more fuel performance data with increased burnup. Duration to achieve targeted burnups depend on ATR operation where lobe powers are adjusted each cycle for the needs of various experiments including PALM cycles. Should PALM cycles occur during AGR-1 irradiation, burnup rates and temperatures are increased briefly during the high power ATR operation.

A tentative schedule indicating major activities for the AGR-1 irradiation test is shown in Figure 8-1.

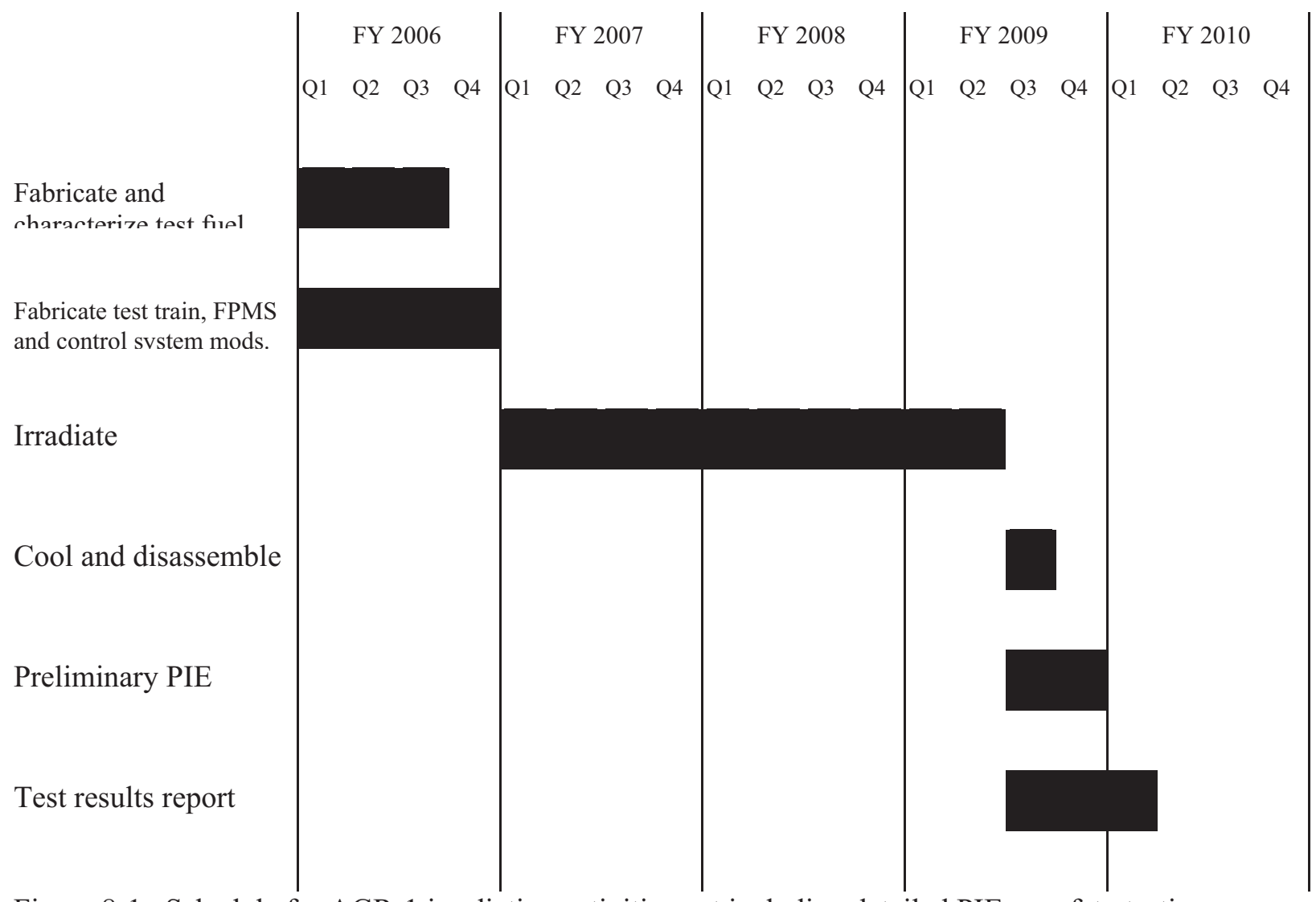

Figure 8-1. Schedule for AGR-1 irradiation activities not including detailed PIE or safety testing. 


\begin{tabular}{|l|lll|} 
AGR-1 Irradiation Experiment Test Plan & Identifier: & INL/EXT-05-00593 \\
& Revision: & 3 & Page: 40 of 41 \\
\hline
\end{tabular}

\section{REFERENCES}

R.G. Ambrosek, “Thermal Analyses for Design of AGR-1”, EDF-5138, October 3, 2005.

R.G. Ambrosek, data files transmitted via e-mail communications to D.L. Knudson and J.T. Maki on January 10 and 18, 2007. Finite element calculations performed with ABAQUS Version 6.6-1 computer program on a SUN W2100z platform.

C. M. Barnes, "AGR-1 Fuel Product Specification and Characterization Guidance", EDF-4380, Rev. 8, April 25, 2006.

BWXT Nuclear Products Division, "G73 Industrial Fuel Fabrication and Development Lot G73D-2069302”, Data Certification Package, March 11, 2005.

G.S. Chang, "Preliminary Physics Analysis of the German UO2 and GA UCO Fuel Test Assembly in North and South Large B Hole Positions", Memorandum GSC-PBMR-04-2002, August 2, 2002.

G.S. Chang and M.A. Lillo, "Confirmatory Neutronics Analysis of the AGR-1 Experiment Irradiated in ATR B-10 Position”, EDF-7120, Rev. 1, February 13, 2007.

J. D. Hunn and R. A. Lowden, "Data Compilation for AGR-1 Baseline Coated Particle Composite LEU01-46T”, ORNL/TM-2006/019, April 2006a.

J. D. Hunn and R. A. Lowden, "Data Compilation for AGR-1 Variant 1 Coated Particle Composite LEU01-47T”, ORNL/TM-2006/020, April 2006b.

J. D. Hunn and R. A. Lowden, "Data Compilation for AGR-1 Variant 2 Coated Particle Composite LEU01-48T”, ORNL/TM-2006/021, May 2006c.

J. D. Hunn and R. A. Lowden, "Data Compilation for AGR-1 Variant 3 Coated Particle Composite LEU01-49T”, ORNL/TM-2006/022, May 2006d.

J.D. Hunn, F.C. Montgomery and P.J. Pappano, "Data Compilation for AGR-1 Baseline Compact Lot LEU01-46T-Z", ORNL/TM-2006/507, August 2006a.

J.D. Hunn, F.C. Montgomery and P.J. Pappano, "Data Compilation for AGR-1 Variant 1 Compact Lot LEU01-47T-Z", ORNL/TM-2006/508, August 2006b.

J.D. Hunn, F.C. Montgomery and P.J. Pappano, "Data Compilation for AGR-1 Variant 2 Compact Lot LEU01-48T-Z”, ORNL/TM-2006/509, August 2006c.

J.D. Hunn, F.C. Montgomery and P.J. Pappano, "Data Compilation for AGR-1 Variant 3 Compact Lot LEU01-49T-Z”, ORNL/TM-2006/510, August 2006d.

J.T. Maki, “AGR-1 Irradiation Test Specification”, EDF-4731, Rev. 1, July 15, 2004.

J.R. Parry, "Fission Heat Rate Reduction From The Use of He-3 Coolant in The AGR-1 Experiment During A PALM Cycle", EDF-7225, Draft September 2006.

D.A. Petti, J.T. Maki, J. Buongiorno, R.R. Hobbins and G.K. Miller, "Key Differences in the Fabrication, Irradiation and Safety Testing of U.S. and German TRISO-coated Particle Fuel and Their Implications on Fuel Performance", INEEL/EXT-02-00300, June 2002.

D.A. Petti, R. Hobbins, J. Kendall and J. Saurwein, "Technical Program Plan for the Advanced Gas Reactor Fuel Development and Qualification Program”, INL/EXT-05-00465, Revision 1, August 2005. 


\begin{tabular}{|l|lll|} 
AGR-1 Irradiation Experiment Test Plan & Identifier: & INL/EXT-05-00593 \\
& Revision: & 3 & \\
& Effective Date: & $10 / 20 / 09$ & Page: 41 of 41 \\
\hline
\end{tabular}

P.B. West, G.A. Marts, E.W. Killian, J.K. Hartwell and S.B. Grover, "Requirements for Design of the Advanced Gas Reactor Experiment AGR-1 for Irradiation in the Advanced Test Reactor", Technical and Functional Requirements, TFR-249, Rev. 1, December 22, 2004.

P.B. West, G.A. Marts, E.W. Killian, J.K. Hartwell and S.B. Grover, "Temperature Control and Off Gas Monitoring Systems for Advanced Gas Reactor Experiment AGR-1", Technical and Functional Requirements, TFR-248, Rev. 1, March 18, 2005. 